\title{
Form factors at strong coupling via a Y-system
}

\author{
Juan Maldacena $^{a}$ and Alexander Zhiboedov ${ }^{b}$ \\ ${ }^{a}$ School of Natural Sciences, Institute for Advanced Study \\ Princeton, NJ 08540, USA \\ ${ }^{b}$ Jadwin Hall, Princeton University \\ Princeton, NJ 08540, USA
}

\begin{abstract}
We compute form factors in planar $\mathcal{N}=4$ Super Yang-Mills at strong coupling. Namely we consider the overlap between an operator insertion and $2 n$ gluons. Through the gauge/string duality these are given by minimal surfaces in $A d S$ space. The surfaces end on an infinite periodic sequence of null segments at the boundary of $A d S$. We consider surfaces that can be embedded in $A d S_{3}$. We derive set of functional equations for the cross ratios as functions of the spectral parameter. These equations are of the form of a Y-system. The integral form of the Y-system has Thermodynamics Bethe Ansatz form. The area is given by the free energy of the TBA system or critical value of Yang-Yang functional. We consider a restricted set of operators which have small conformal dimension compared to $\sqrt{\lambda}$.
\end{abstract}




\section{Contents}

1. Introduction . . . . . . . . . . . . . . . . . . . . . . . . . . . . . . . . 2

2. Short review of the strong coupling prescription for computing form factors . . . . . . 5

3. Derivation of the Y-system for general monodromy . . . . . . . . . . . . . . . . . 6

3.1. Preliminaries . . . . . . . . . . . . . . . . . . . . . . . . . . . . 6

3.2. Kinematics of the general problem . . . . . . . . . . . . . . . . . . . . . 11

3.3. Truncation of the Y-system . . . . . . . . . . . . . . . . . . . . . . . . . 12

3.4. Normalization independent definition of the Y-functions . . . . . . . . . . . . . 13

4. Constant monodromies . . . . . . . . . . . . . . . . . . . . . . . . . . . . . 15

4.1. Recovering the Y-system for scattering amplitudes . . . . . . . . . . . . . . . 16

4.2. Y-system for the form factors of operators with small dimension . . . . . . . . . . 16

4.3. $Y$-system for $Z_{m}$ symmetric polygons . . . . . . . . . . . . . . . . . . . . . 17

5. Y-system for the form factors . . . . . . . . . . . . . . . . . . . . . . . . . . 18

5.1. Zig-zag solution . . . . . . . . . . . . . . . . . . . . . . . . . . . . . . 18

5.2. Analytic properties of the Y-functions . . . . . . . . . . . . . . . . . . . . 21

5.3. Evaluating Y's . . . . . . . . . . . . . . . . . . . . . . . . . . . . . . 22

5.4. Integral form of the equations . . . . . . . . . . . . . . . . . . . . . . . . 24

6. Area in the case of form factors . . . . . . . . . . . . . . . . . . . . . 25

6.1. General formula . . . . . . . . . . . . . . . . . . . . . . . . . . 25

6.2. Area as the free energy . . . . . . . . . . . . . . . . . . . . . . . . . . . 26

6.3. Area as the critical value of Yang-Yang functional . . . . . . . . . . . . . . . 26

7. Exact solutions . . . . . . . . . . . . . . . . . . . . . . . . . . . . . . . . 27

7.1. High temperature limit of the form factor Y-system . . . . . . . . . . . . . . . 28

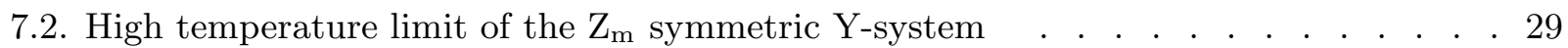

7.3. Exact solution for the 4 -cusp form factor . . . . . . . . . . . . . . . . . . . 30

8. Conclusions . . . . . . . . . . . . . . . . . . . . . . . . . . . 33

9. Acknowledgments . . . . . . . . . . . . . . . . . . . . . . . . . . . . . . . 34

Appendix A. Derivation of the monodromy . . . . . . . . . . . . . . . . . . . . . 34

Appendix B. $\mathrm{Z}_{\mathrm{m}}$ symmetric polygons . . . . . . . . . . . . . . . . . . . . . . . . 36

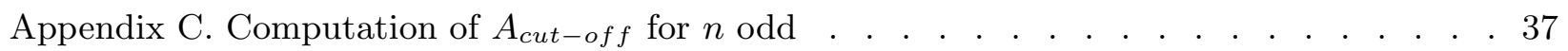

Appendix D. Integral equations for complex masses . . . . . . . . . . . . . . . . . . 39

Appendix E. Calculation of $N=4 k$ gluon amplitudes by taking the double soft limit . . . 39

E.1. Soft limit of the $A_{0}$. . . . . . . . . . . . . . . . . . . . . . . . . . . . 42

E.2. Soft limit of the $A_{B D S-l i k e}-A_{B D S}$. . . . . . . . . . . . . . . . . . . . . 42

E.3. The octagon . . . . . . . . . . . . . . . . . . . . . . . . . . . . . . . . 45

Appendix F. On the connection with BTZ black holes . . . . . . . . . . . . . . . . 46

Appendix G. The monodromy in terms of coordinates . . . . . . . . . . . . . . . . . 4 


\section{Introduction}

Recently there has been considerable progress in the calculation of light-like Wilson loops both at weak and strong coupling. These are Lorentzian objects that depend on a finite number of parameters, namely positions of the cusps. One of the main motivations to calculate such objects was the fact that they describe color-ordered scattering amplitudes

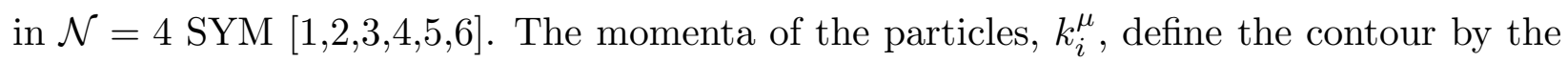
simple rule $x_{i}^{\mu}-x_{i+1}^{\mu}=k_{i}^{\mu}$. However, very soon it became clear that light-like Wilson loops are related to broader class of objects, namely to form factors, non-supersymmetric Wilson loops and correlation functions [7, 8, 9, 10].

At strong coupling, the problem can be solved by using the integrability of classical strings on $A d S$ [11, 12, 13]. The key steps were: first, introduce a family of flat connections parameterized by an arbitrary spectral parameter $\mathcal{A}\left(\zeta=e^{\theta}\right)$. With these flat connections one can consider the flat section problem which allows one to introduce a set of the spectral parameter dependent cross ratios $Y_{k}(\theta)$. Then one finds a set of functional equations which constraints the $\theta$ dependence of Y-functions. The system is specified with boundary conditions at $\theta \rightarrow \pm \infty$ which are obtained through a WKB analysis of the flat section problem. Then one can derive an integral form of these equations. These are of a TBA form [14, 15, 16, 17]. The non-trivial part of the area is given by the free energy of that TBA system which depends on a set of mass parameters in terms of which the kinematics is encoded. It is also convenient to have an expression for the area that depends only on the kinematic information, namely cross ratios. This can be obtained by solving for the mass parameters in terms of the physical cross ratios. The final area is given by the critical value of Yang-Yang functional [8].

In this paper we consider a more general problem which corresponds to the additional insertion of a closed string state on the worldsheet. At the insertion point, $z_{0}$, the connection $\mathcal{A}\left(z_{0}\right)$ exhibits specific singularity. The inserted state is specified by the monodromy $\Omega(\zeta)$ of the flat connection around the insertion point [18,19].

Physically, we are studying processes involving a local operator and a fixed number of particles. Such objects are called form factors. The operator creates a state which is then projected on to a state with a definite number of gluons. This is a problem that is common in QFT. For example, one can have in mind all processes like $e^{+} e^{-} \rightarrow$ jets to lowest order in $\alpha_{e m}$ but to all orders in $\alpha_{s}$. Here the electromagnetic current creates a QCD state which we want to study. 


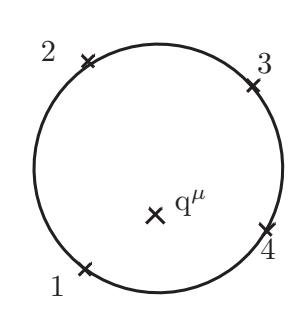

(a)

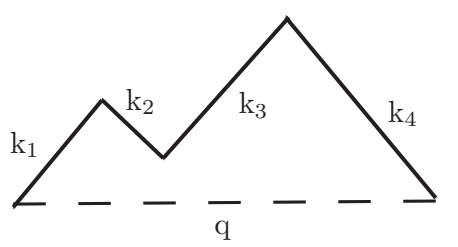

(b)

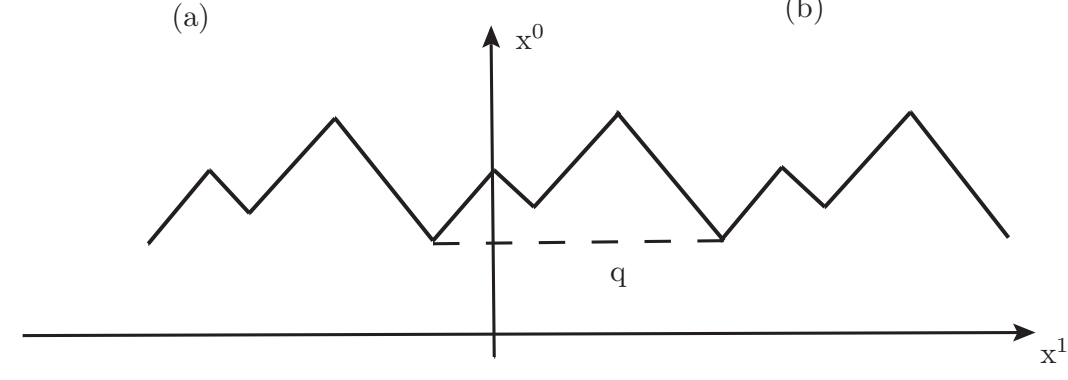

(c)

Fig. 1: In (a) we show a worldsheet with topology of a disc. It has open string insertions at the boundary corresponding to the gluons and a closed string insertion corresponding to an operator with momentum $q^{\mu}$. In (b) we show the kinematics of the process. Each gluon has a light-like momentum. The operator has an arbitrary momentum, for convenience we choose it to be spacelike and collinear to $\mathrm{x}^{1}$ axis. In (c) we show the periodic configuration which arises from the configuration (b) after we perform the T-duality transformation. Note that the doted line in (b) is not part of the contour of the Wilson loop.

At strong coupling the problem involves computing the area of a certain minimal surface. The geometric problem is obtained after performing a certain T-duality, as explained in [7]. The surfaces end on an infinite periodic sequence of null segments at the boundary of $A d S$ space. The period is defined by the momentum, $q^{\mu}$, of the inserted operator. The shape of the polygon within each period is defined by momenta of gluons, see fig. 1. Another manifestation of the presence of the operator comes from the fact that we consider solutions that go to the horizon.

We consider surfaces that can be embedded in $A d S_{3}$. This amounts to considering $R^{1,1}$ kinematics in dual field theory language 1 . We extend the approach of [13 to cases with general monodromy and derive a set of functional equations for the conformal cross as functions of the spectral parameter. Then we study several cases when the monodromy

1 Remember that we limit the kinematics of the external gluons. Gluons propagating in the loops live in $R^{1,3}$. 
is spectral parameter independent. In the case of trivial monodromy they reduce to the Y-system for scattering amplitudes.

We then consider form factors defined via

$$
\left\langle\left\{k_{i}^{\text {in }}\right\}|\mathcal{O}(q)|\left\{k_{j}^{\text {out }}\right\}\right\rangle=\int d^{4} x e^{i q x}\left\langle\left\{k_{i}^{\text {in }}\right\}|\mathcal{O}(x)|\left\{k_{j}^{\text {out }}\right\}\right\rangle .
$$

We consider operators with conformal dimensions small compared to $\sqrt{\lambda}$ 2 . The problem boils down to studying strings in massless BTZ black hole geometry [20]. This geometry is simply $A d S_{3}$ with the identification $x \sim x+q$ where $x$ is space Poincare coordinate see fig. 1. Originally the prescription for calculating such objects was found in [7]. At weak coupling these objects were considered in [21].

Here we analyze this case in detail and find all pieces of the area. The most complicated one is given by the free energy of the TBA system or as the critical value of Yang-Yang functional. We get a result that is independent of the operator that we insert (as long as they have small dimension compared to $\sqrt{\lambda}$ ). One can understand it qualitatively as follows: at strong coupling there is copious production of very low energy gluons, so the emission of small number of gluons is equally strongly disfavored for all operators [22, 23]. The dependence on the operator, and on the polarization of the gluons, should reappear at one loop in the $1 / \sqrt{\lambda}$ expansion.

Our paper is organized as follows. In section two we review [7]. In section three we derive the Y-system for a general monodromy. This is a general discussion which applies to cases that are more general than what we discuss later. In section four we analyze the case when the monodromy does not depend on the spectral parameter. In section five we concentrate on Wilson loops in a massless BTZ background and derive integral form of the equations which are of the TBA form. The most non-trivial part of the area is given by the free energy of the corresponding TBA system or critical value of Yang-Yang functional. In section six we analyze all pieces of the area. In section seven we consider exact solutions of the Y-system which allows us to check our formulas. In addition we derive the explicit answer for the case of an operator going into four gluons. We end with conclusions. We include several appendices with useful technical details. In particular, we explain how one can compute the area when the number of gluons is proportional to 4 by taking the double soft limit, both for amplitudes and for the case of form factor (this was also considered in [24]).

2 Examples of such operators are the stress tensor, the R-currents, any BPS operator, low level massive string states with dimensions $\propto \lambda^{1 / 4}$. This excludes operators corresponding to semiclassical string states that have energies going like $\lambda^{1 / 2}$. 


\section{Short review of the strong coupling prescription for computing form factors}

Here we briefly recall the prescription for calculation of the form factors at strong coupling [7].

We are working in the $A d S_{5}$ space describing the gravity dual to $\mathcal{N}=4 \mathrm{SYM}$ with the metric

$$
d s^{2}=\frac{d x^{2}+d z^{2}}{z^{2}}
$$

where $x^{\mu}$ are the coordinates of the $R^{1,3}$ space where the field theory lives.

Since scattering amplitudes in CFT's are ill-defined one needs to introduce IR regulator. A convenient regulator consists of D-branes which are located at $z_{I R}$. Gluons are open strings ending on them. Removing the regulator corresponds to sending $z_{I R} \rightarrow \infty$. After we introduced the regulator we can consider complex classical solutions of the string equations of motion whose boundary conditions in the past and future infinity are set at $z=z_{I R}$ where asymptotic gluons live. In addition, the worldsheet reaches $z=0$ where the operator is inserted.

To describe these classical solutions it is very convenient to perform a T-duality transformation along the four worldvolume directions and to make the change of variables $r=\frac{1}{z}$. This transformation leaves the metric invariant and exchanges the momenta by "windings".

In the new coordinates the gluon states are represented by a sequence of light-like lines $k_{i}^{\mu}$ at $r=0$. However because of the presence of the operator this sequence is not closed, namely we have $\sum_{i=1}^{2 n} k_{i}^{\mu}=q^{\mu}$ where $q^{\mu}$ is the operator momentum. The operator is represented by a closed string so we should identify the ends of the sequence which is the same as compactifying the coordinate along $q^{\mu}$ and considering a closed string winding this coordinate. Equivalently we can unfold this picture and consider an infinite periodic set of light-like segments given by momenta $k_{i}^{\mu}$. For the case of $A d S_{3}$ which will be in the focus of the paper this procedure is illustrated in fig. 1. The operator which was inserted at $z=0$, now leads to a string worldsheet which goes to $r=\infty$.

After one finds the solution that minimizes the area with given boundary condition the form factor takes the form

$$
\left\langle\left\{k_{i}^{\text {in }}\right\}|\mathcal{O}(q)|\left\{k_{j}^{\text {out }}\right\}\right\rangle=e^{-\frac{\sqrt{\lambda}}{2 \pi}(\text { Area })_{T}} \mathrm{~F}_{1}\left(1+\frac{1}{\sqrt{\lambda}} \mathrm{F}_{2}+\cdots\right)
$$

where the leading term $(\text { Area })_{T}$ is the area of one period and its computation is the main topic of this paper. $F_{1}$ is the one loop correction and would contain information about the polarizations and the particular operator we are considering. $F_{2}$ is the two loop correction in the $1 / \sqrt{\lambda}$ expansion. 


\section{Derivation of the $\mathrm{Y}$-system for general monodromy}

We consider minimal surfaces that can be embedded in $A d S_{3}$ subspace of $A d S_{5}$. From the field theory side it corresponds to gluons and operators with momenta lying in an $R^{1,1}$ subspace of $R^{1,3}$. Throughout this paper we consider a problem with $2 n$ gluons. We first consider $n$ odd, which simplifies the consideration. We then get the answer for $n$ even by taking double soft limit at the very end.

\subsection{Preliminaries}

We are interested in the classical dynamics of strings in $A d S_{3}$ space. $A d S_{3}$ can be conveniently written as the following surface in $R^{2,2}$

$$
Z_{-1}^{2}+Z_{0}^{2}-Z_{1}^{2}-Z_{2}^{2}=1
$$

We use the Poincare coordinates

$$
x^{ \pm}=\frac{Z_{1} \pm Z_{0}}{Z_{-1}+Z_{2}}, \quad r=\frac{1}{Z_{-1}+Z_{2}}
$$

to define the kinematics of the process.

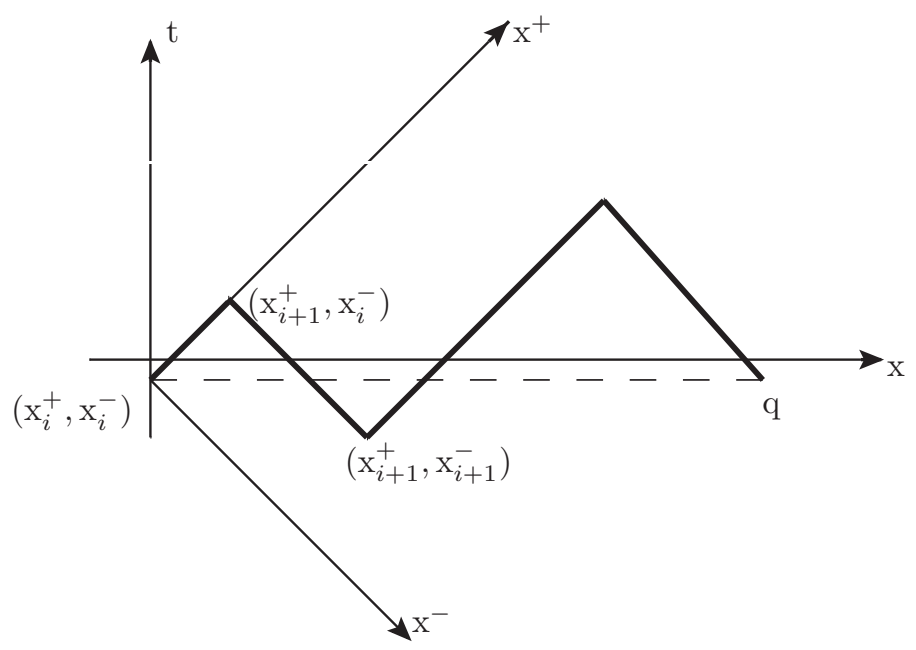

Fig. 2: The labeling of cusps at the boundary of $A d S_{3}$. The $q$ stands for the operator momentum and it is the period of the polygon at the same time. 
For the distance between two points on the boundary we use the following notation

$$
x_{i j}^{ \pm}=x_{i}^{ \pm}-x_{j}^{ \pm}
$$

and we label the cusps as it shown at fig. 2 .

A point in $A d S_{3}$ space can be conveniently written as an $S L(2, R)$ group element

$$
Z_{a \dot{a}}=\left(\begin{array}{cc}
Z_{-1}+Z_{2} & Z_{1}-Z_{0} \\
Z_{1}+Z_{0} & Z_{-1}-Z_{2}
\end{array}\right)_{a \dot{a}}
$$

The $S O(2,2)$ symmetry of the model is realized by left and right multiplication by $S L(2, R) \times S L(2, R)$. We are interested in calculation of the divergent integral, we use the notation of [1],

$$
A=\int d x d y \partial_{i} Z \partial_{i} Z=4 \int d^{2} z e^{2 \alpha}=2 \int d^{2} z \operatorname{Tr}\left[\Phi_{z} \Phi_{\bar{z}}\right] .
$$

The function $\alpha$ obeys the generalized Sinh-Gordon equation [25,26]

$$
\partial \bar{\partial} \alpha-e^{2 \alpha}+|p(z)|^{2} e^{-2 \alpha}=0 .
$$

Here $p(z)=\frac{1}{2} \operatorname{Tr}\left[\Phi_{z}^{2}\right]$ is a holomorphic function in which the kinematics of the process is encoded. This equation should be supplemented with appropriate boundary conditions for $\alpha$.

The problem of finding the a minimal area surface embedded in $A d S_{3}$ can be formulated in terms of a $\mathrm{Z}_{2}$ projection of an $S U(2)$ Hitchin system where $\Phi$ is the Higgs field [27]. The $Z_{2}$ projection acts on fields as follows $\Phi_{z}=-\sigma_{3} \Phi_{z} \sigma_{3}, \Phi_{\bar{z}}=-\sigma_{3} \Phi_{\bar{z}} \sigma_{3}, A=\sigma_{3} A \sigma_{3}$.

We study the flat sections, $\psi$, that, by definition, obey the equation

$$
(d+\mathcal{A}) \psi=0
$$

with

$$
\mathcal{A}=\frac{\Phi_{z} d z}{\zeta}+A+\zeta \Phi_{\bar{z}} d \bar{z}
$$

here $\zeta$ is the spectral parameter and the original surface can be read off from the solution of (3.7) at $\zeta=1, i$.

Recall that the worldsheet is parameterized by the whole complex plane $z$. In the large $z$ region some of the flat sections diverge. This means that worldsheet goes to the 
boundary of $A d S$ space. The fact that the worldsheet goes to different cusps is due to Stokes phenomena.

At large $z$ it is possible to diagonalize the Higgs fields $\Phi(z) \sim \sqrt{p} \cdot \operatorname{diag}(1,-1), \Phi(\bar{z}) \sim$ $\sqrt{\bar{p}} \cdot \operatorname{diag}(1,-1)$. This allows one to determine the form of two independent solutions of the section problem (3.7) at large $z$

$$
\psi_{a} \sim \exp \left((-1)^{a} \frac{\int d z \sqrt{p(z)}}{\zeta}+(-1)^{a} \zeta \int d \bar{z} \sqrt{\bar{p}(\bar{z})}\right), \quad a=0,1
$$

The number of Stokes sectors is determined by the number of cusps or equivalently by the degree of polynomial. Within each Stokes sector the worldsheet reaches a different point at the boundary of $A d S$. It is very useful to define the smallest solution $s_{i}$ in each of the Stokes sectors. It is a flat section which has fastest decay rate as $z \rightarrow \infty$. This solution exists and it is unique in each Stokes sector, up to an overall rescaling.

Importantly, in the case of an operator insertion we assume that the connection has a singularity at $z=0$, which corresponds to the operator insertion. The operator is specified by the monodromy, $\Omega(\zeta)$, of the connection around $z=0$.

We are going to study the problem at large $z$ with this monodromy which geometrically corresponds to certain periodic Wilson loop. Importantly, the connection is single-valued and comes to itself when we go around the insertion point $z \rightarrow z e^{2 \pi i}$. However due to the non-trivial monodromy, the sections do not go to themselves and the Wilson polygons are not closed.

Let us consider the small solution in the $i$ 'th Stokes sector (in some terminology these are anti-Stokes sector)

$$
\left(i-\frac{3}{2}\right) \frac{2 \pi}{n}+\frac{2}{n} \operatorname{Arg}[\zeta]<\operatorname{Arg}[z]<\left(i-\frac{1}{2}\right) \frac{2 \pi}{n}+\frac{2}{n} \operatorname{Arg}[\zeta]
$$

which is denoted by $s_{i}(\zeta)$. Due to $Z_{2}$ projection $\sigma_{3} s_{i}\left(e^{i \pi} \zeta\right)$ is also a solution of the flat section problem. From the large $z$ form of the solution one can easily see that it is a small solution in $(i+1)$ 'th Stokes sector. Thus, we can define $s_{i+1}(\zeta)=i \sigma_{3} s_{i}\left(e^{i \pi} \zeta\right)$ and normalize $\left\langle s_{1} s_{2}\right\rangle=1$. From the definition it follows that

$$
\left\langle s_{i} s_{j}\right\rangle\left(e^{i \pi} \zeta\right)=\left\langle s_{i+1} s_{j+1}\right\rangle(\zeta)
$$

Taking into account the normalization we chose it leads to

$$
\left\langle s_{i} s_{i+1}\right\rangle=1 \text {. }
$$

Throughout the paper we extensively use $S L(2)$ invariant inner product for flat sections defined as

$$
\left\langle\psi_{a} \psi_{b}\right\rangle=\psi_{a} \wedge \psi_{b}=\epsilon^{\alpha \beta} \psi_{\alpha, a} \psi_{\beta, b}
$$




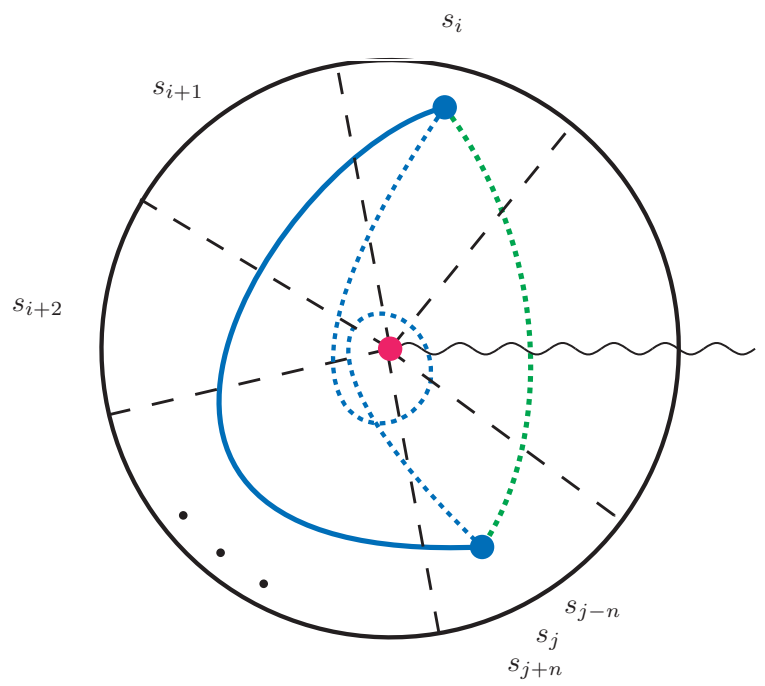

Fig. 3: The $z$ plane with Stokes sectors separated by black dotted lines. In each Stokes sector we have a unique small solution. Starting from 0-th sector we have infinite chain $\ldots, s_{-2}, s_{-1}, s_{0}, s_{1}, \ldots$ of small solutions . To evaluate the inner product between the small solutions $s_{i}$ and $s_{j}$ with $i<j$ we need to specify a path of analytic continuation from the Stokes sector where small solutions are defined to the point where the inner product is evaluated. We choose it to be unfolded anti-clockwise path that connects sectors $i$ and $j$ which is given by solid blue line. The red dot corresponds to the operator insertion. The green dotted line indicates the one that we use to evaluate $\left\langle s_{i} s_{j-n}\right\rangle$. The blue dotted one serves to evaluate $\left\langle s_{i} s_{j+n}\right\rangle$. The wavy line allows one easily count the number of correspondent Stokes sector with correspondent small solution. In the case when there is no operator insertion $s_{i+n} \sim s_{i}$ and all paths are equivalent.

This product does not depend on the point $z$ where we evaluate it. However, it can depend on the path that we choose to go from the region at infinity where $s_{i}$ are naturally defined to the point where we evaluate it. In order to define the inner product in unique way we need to specify this path. It is convenient to go to the covering space of the punctured disk, so that we think of $z$ and $e^{2 \pi i} z$ as different points. The sections defined above are not periodic on the punctured disk but they are naturally defined on the covering space. Note also that the sections $s_{i}$ defined as the ones small in the sector (3.10) are really defined on the covering space. In other words, the sectors in (3.10) are the sectors of the covering space. There is an infinite number of such sectors. When we take inner products, such as (3.13) we need to transport the sections to a common point. We do this transport in the covering space, which is simply connected. Of course, once we project down to the disk, it might happen that the path that we follow to evaluate an inner product might wind around $z=0$ a number of times. This can be seen in fig. 3 . In the covering space, 


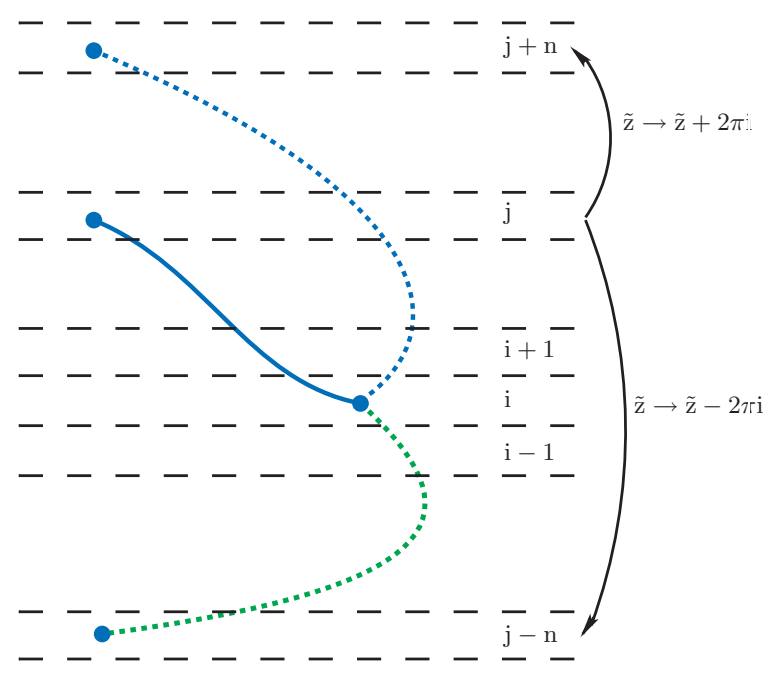

Fig. 4: We represent the same as in fig. 3 but in the covering space, the $\tilde{z}$ plane. The two spaces are related through the map $z=e^{\tilde{z}}$. Here the boundary of the disk of fig. 3 is to the right. The origin in fig. 3 is to the left. The lines represent the same inner products as in fig. 3 .

the same figure looks as in fig. 4. So, for example, if we evaluate $\left\langle s_{0} s_{1}\right\rangle$ we do not wind around $z=0$. However, if we evaluate $\left\langle s_{n} s_{0}\right\rangle$ then we wind once around $z=0$. Note that $s_{0}$ and $s_{n}$ are not the same section. $s_{n}$ is the section that is small after we have gone around $z=0$ once.

One can use the small solutions to define conformal cross ratios as functions of the spectral parameter as follows

$$
\chi_{i j k l}(\zeta)=\frac{\left\langle s_{i} s_{j}\right\rangle\left\langle s_{k} s_{l}\right\rangle}{\left\langle s_{i} s_{k}\right\rangle\left\langle s_{j} s_{l}\right\rangle} .
$$

Notice that these objects are independent of normalization of small solutions. The kinematics of the process is then encoded in

$$
\chi_{i j k l}(1)=\frac{x_{i j}^{+} x_{k l}^{+}}{x_{i k}^{+} x_{j l}^{+}}, \quad \chi_{i j k l}(i)=\frac{x_{i j}^{-} x_{k l}^{-}}{x_{i k}^{-} x_{j l}^{-}}
$$

We can derive functional equations for these objects as explained in [13]. We use Schouten identity

$$
\left\langle s_{i} s_{j}\right\rangle\left\langle s_{k} s_{l}\right\rangle+\left\langle s_{i} s_{l}\right\rangle\left\langle s_{j} s_{k}\right\rangle+\left\langle s_{i} s_{k}\right\rangle\left\langle s_{l} s_{j}\right\rangle=0
$$

and the definition $T_{k}=\left\langle s_{0} s_{k+1}\right\rangle\left(e^{-i(k+1) \frac{\pi}{2}} \zeta\right)$ to get the Hirota equation

$$
T_{k}^{+} T_{k}^{+}=T_{k+1} T_{k-1}+1
$$


where $f^{ \pm}=f\left(e^{ \pm i \frac{\pi}{2}} \zeta\right)$ and 3.12 were used. Recall that from the definition and normalization convention it follows that $T_{-1}=0$ and $T_{0}=1$. As the next step we introduce the Y-functions $Y_{s}(\zeta)=T_{s-1} T_{s+1}$ which contain all the kinematic information of the problem. They are equal to conformal cross ratios at particular values of $\zeta$. In terms of the Y-functions we get the set of equations

$$
Y_{s}^{+} Y_{s}^{-}=\left(1+Y_{s+1}\right)\left(1+Y_{s-1}\right) ; \quad Y_{0}=0 .
$$

This set of equations is truncated from below because $Y_{0}$ is equal to zero according to its definition. The equations will be truncated from above by the periodicity condition, as we will explain below. The precise condition depends on $\Omega(\zeta)$.

\subsection{Kinematics of the general problem}

Let's count the number of parameters we have in the problem. On the boundary of $A d S$ we have a polygonal Wilson loop which is periodic up to the action of the two copies of $S L(2, R)$.

To be more precise we are thinking about the Wilson polygon with an infinite number

of cusps at the positions $\left(x_{i}^{+}, x_{i}^{-}\right)$. However the position of the cusp $\left(x_{i+n}^{+}, x_{i+n}^{-}\right)$is related to the position of $\left(x_{i}^{+}, x_{i}^{-}\right)$through a conformal transformation

$$
Z_{i+n}=\hat{\Omega}_{L} Z_{i} \hat{\Omega}_{R}
$$

Of course, these conformal transformations are the monodromies around the origin. If we concentrate on $x^{+}$then the Wilson loop is periodic up to the action of $S L(2, R)$ transformation. Let us ask how many independent cross ratios we can make with these constraints.

By conformal transformation we can fix the points $x_{i}^{+}, i=0,1,2$. Then we can have arbitrary points $x_{i}^{+}, i=3, . ., n+2$ where points $i=n, n+1, n+2$ specify the monodromy. In other words, the monodromy is the unique conformal transformation which maps the points $0,1,2$ to the points $n, n+1, n+2$.

Thus, including the degrees of freedom necessary to specify the monodromy we have $2 n$ variables.

This counting is just a property of the polygon at the boundary and does not depend on the object inserted in the interior of the worldsheet. 


\subsection{Truncation of the $Y$-system}

At the level of flat sections we introduce the monodromy matrix as follows

$$
\left(\begin{array}{l}
s_{1} \\
s_{0}
\end{array}\right)\left(z e^{2 \pi i}, \zeta\right)=\Omega(\zeta)\left(\begin{array}{l}
s_{1} \\
s_{0}
\end{array}\right)(z, \zeta), \quad\left\langle s_{0} s_{1}\right\rangle=1
$$

Recall that any two linearly independent solutions of the flat section problem form a basis and here we choose $s_{0}$ and $s_{1}$ as basis solutions. $\Omega(\zeta)$ can be though as being known and characterizing the operator.

By definition $s_{n}(z, \zeta)$ is the solution which is small in the same sector as $s_{0}(z, \zeta)$ but after going around the complex plane once. So it should be proportional to $s_{0}\left(z e^{-2 \pi i}, \zeta\right)$

$$
s_{n}(z, \zeta)=B(\zeta) s_{0}\left(z e^{-2 \pi i}, \zeta\right)
$$

where we used the fact that the connection is single-valued.

Here $B(\zeta)$ appears due to the normalization convention and it is obviously different from zero for any $\zeta$. Changing the spectral parameter and multiplying by $i \sigma_{3}$ we get

$$
s_{n+1}(z, \zeta)=B^{++}(\zeta) s_{1}\left(z e^{-2 \pi i}, \zeta\right)
$$

If we take the wedge of $(3.20)$ and $(3.21)$ we get

$$
B^{+} B^{-}=1
$$

Using these definitions we have

$$
\left(\begin{array}{c}
B s_{n+1} \\
B^{-1} s_{n}
\end{array}\right)(z, \zeta)=\left(\begin{array}{l}
s_{1} \\
s_{0}
\end{array}\right)\left(z e^{-2 \pi i}, \zeta\right)=\Omega^{-1}(\zeta)\left(\begin{array}{l}
s_{1} \\
s_{0}
\end{array}\right)(z, \zeta)
$$

Taking the wedge we can calculate the trace of the monodromy

$$
B(\zeta)\left\langle s_{0} s_{n+1}\right\rangle(\zeta)-B^{-1}(\zeta)\left\langle s_{1} s_{n}\right\rangle(\zeta)=\operatorname{Tr}\left[\Omega^{-1}(\zeta)\right]=\operatorname{Tr}[\Omega(\zeta)]
$$

here we used the fact that $\operatorname{det}[\Omega]=1$ which immediately follows from the fact that the connection (3.8) is traceless. Using the definition for $T$ functions, (3.24) can be rewritten as

$$
B(\tilde{\zeta}) T_{n}(\zeta)-B^{-1}(\tilde{\zeta}) T_{n-2}(\zeta)=\operatorname{Tr}[\Omega(\tilde{\zeta})]
$$


where $\tilde{\zeta}=e^{-i(n+1) \pi / 2} \zeta$. Using this equation we can truncate the chain of Hirota equations by expressing $T_{n}$ through $T_{n-2}$

$$
T_{n}(\zeta)=B^{-1}(\tilde{\zeta}) \operatorname{Tr}[\Omega(\tilde{\zeta})]+B^{-2}(\tilde{\zeta}) T_{n-2}(\zeta)=B^{-1}(\tilde{\zeta})[\operatorname{Tr}[\tilde{\Omega}]+\bar{Y}]
$$

where we introduced

$$
\bar{Y}(\zeta)=B^{-1}(\tilde{\zeta}) T_{n-2}(\zeta), \quad \operatorname{Tr}[\tilde{\Omega}]=\operatorname{Tr} \Omega(\tilde{\zeta})
$$

Using $B^{+} B^{-}=1$, we get the following Y-system for the general periodic Wilson loop

$$
\begin{aligned}
Y_{s}^{+} Y_{s}^{-} & =\left(1+Y_{s+1}\right)\left(1+Y_{s-1}\right), \quad s=1, \ldots, n-3 \\
Y_{n-2}^{+} Y_{n-2}^{-} & =\left(1+Y_{n-3}\right)\left(1+\operatorname{Tr}[\tilde{\Omega}] \bar{Y}+\bar{Y}^{2}\right) \\
\bar{Y}^{+} \bar{Y}^{-} & =1+Y_{n-2} .
\end{aligned}
$$

Here the truncation condition was used to get the equation for $Y_{n-2}$. We started with $Y_{n-2}^{+} Y_{n-2}^{-}=\left(1+Y_{n-3}\right)\left(1+Y_{n-1}\right)$ and then used the fact that $Y_{n-1}=T_{n-2} T_{n}=$ $T_{n-2} B^{-1}[\operatorname{Tr}[\Omega(\tilde{\zeta})]+\bar{Y}]=\operatorname{Tr}[\tilde{\Omega}] \bar{Y}+\bar{Y}^{2}$.

Note that due to the $\mathrm{Z}_{2}$ projection the trace of the monodromy $\operatorname{Tr}[\tilde{\Omega}]=\operatorname{Tr}[\Omega(\zeta)]$ for $n$ odd. For $n$ even, it is simply $\operatorname{Tr}[\tilde{\Omega}]=\operatorname{Tr}[\Omega(\zeta)]^{+}$.

Of course, to choose the solution one needs to supplement these equations with the analytic properties of Y-functions. Another issue is to relate the solution to the expression for the area.

We immediately see that the number of cross ratios that appear in the system is $2(n-1)$. Also we need to specify $\operatorname{Tr}[\tilde{\Omega}]$ at $(\zeta=1 ; i)$ to specify the polygon at the boundary. Thus, including the monodromy we have $2 n$ parameters which agrees with the general counting above.

\subsection{Normalization independent definition of the $Y$-functions}

In the derivation above we use one particular normalization of small solutions. It is convenient to write the $Y$ functions also for general normalizations. For $Y_{s}$ this is identical to the case of scattering amplitudes [13]

$$
\begin{aligned}
Y_{2 k} & =\frac{\left\langle s_{-k}, s_{k}\right\rangle\left\langle s_{-k-1}, s_{k+1}\right\rangle}{\left\langle s_{-k-1}, s_{-k}\right\rangle\left\langle s_{k}, s_{k+1}\right\rangle} \\
Y_{2 k+1} & =\left(\frac{\left\langle s_{-k-1}, s_{k}\right\rangle\left\langle s_{-k-2}, s_{k+1}\right\rangle}{\left\langle s_{-k-2}, s_{-k-1}\right\rangle\left\langle s_{k}, s_{k+1}\right\rangle}\right)^{+} .
\end{aligned}
$$


Notice that to get physical values for cross ratios one needs to evaluate $Y_{2 k}(\zeta=1, i)$ and $Y_{2 k+1}(\zeta=1, i)$ correspondingly 3 . One can think of points at the boundary of $A d S$ space as given by points, $\hat{Z}$, in $R^{2,2}$ with the constraint $\hat{Z}^{2}=0$ and the identification $\hat{Z} \sim \lambda \hat{Z}$. Then one can solve the constraint $\hat{Z}^{2}=0$ introducing a pair of spinors $\lambda, \tilde{\lambda}$, via $\hat{Z}_{a \dot{b}}=\lambda_{a} \tilde{\lambda}_{\dot{b}}$. Where the $a$ index transforms under the left $S L(2)$ and the $\dot{b}$ index under the right $S L(2)$. These are very simply related to $x^{ \pm}$coordinates, $x^{+}=\frac{\lambda_{2}}{\lambda_{1}}, x^{-}=\frac{\tilde{\lambda}_{2}}{\tilde{\lambda}_{1}}$. As explained in [13] we can rewrite the cross ratios in terms of the positions of the cusps by replacing the $s_{i}$ in (3.29) by $\lambda$ or $\tilde{\lambda}$. Thus the physical values of the Y-functions correspond to the points of the unfolded infinite, periodic, polygons.

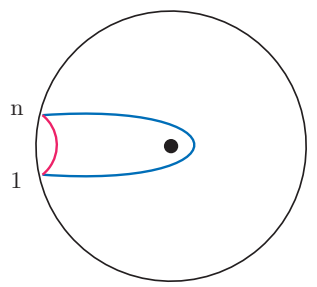

Fig. 5: Inner products in the computation of $\bar{Y}$. We have two consecutive small sections on the disk. We take one inner product going around the origin (in blue) and we divide by the same inner product but defined on the more direct path (in red).

The new element in the case of the operator insertion is $\bar{Y}$. We calculate $B$ by taking the inner product of (3.20) with $\tilde{s}_{1}=s_{1}\left(z e^{-i 2 \pi}\right)$. We then get

$$
\bar{Y}(\zeta)=\frac{\left\langle s_{1} s_{n}\right\rangle}{\left\langle s_{n} \tilde{s}_{1}\right\rangle}\left(\zeta e^{-i \pi \frac{n+1}{2}}\right)=-\frac{\left\langle s_{1} s_{n}\right\rangle}{\left\langle s_{1} s_{n}\right\rangle_{\text {Other Contour }}}\left(\zeta e^{-i \pi \frac{n+1}{2}}\right)
$$

Where the "Other Contour" is simply a contour which connects the two cups without going around the origin. It is the same contour we would use to evaluate $\left\langle s_{0} s_{1}\right\rangle$. A simple graphical representation of $\bar{Y}$ is in fig. 5 .

The expression (3.30) defines $\bar{Y}$ in a normalization independent fashion, but it is not yet defined in terms of target space quantities that specify the shape of the polygon. In other words, we would like to derive an expression in terms of the $\lambda_{i}$ and $\tilde{\lambda}_{i}$ which specify the target space contour. This can easily be done. We can derive the expression

$$
\bar{Y}\left(\zeta=i^{n+1}\right)=-\frac{\left\langle\lambda_{1}, \lambda_{n}\right\rangle}{\left\langle\hat{\Omega} \lambda_{1}, \lambda_{n}\right\rangle}
$$

3 In general, all $Y_{s}\left(\zeta=i^{k}\right)$ are physical cross ratios. The ones we mentioned are a particular set of functionally independent ones. 
where $\hat{\Omega}$ is the $S L(2, R)$ transformation that is uniquely determined by the condition that it (projectively) sends points $\lambda_{0}, \lambda_{1}, \lambda_{2}$ to $\lambda_{n}, \lambda_{n+1}, \lambda_{n+2}$. Then all the $\lambda_{i}$ obey, $\lambda_{n+i} \propto \hat{\Omega} \lambda_{i}$. We have a similar expression in terms of $\tilde{\lambda}_{i}$ and the value of $\bar{Y}$ at a shifted value of $\zeta$.

Alternatively, we can solve for $\hat{\Omega}$ in terms of the $\lambda$ 's and write an expression which only involves the target space positions of the points (see Appendix G)

$$
\begin{aligned}
\Lambda^{2} & =\frac{x_{n+2, n+1}}{x_{2,1}} \frac{x_{2,0}}{x_{n+2, n}} \frac{x_{n+1, n}}{x_{1,0}}, \\
\bar{Y}\left(\zeta=i^{n+1}\right) & =\frac{x_{n, 1}^{+}}{x_{n+1, n}^{+}} \Lambda^{+}, \\
\bar{Y}\left(\zeta=i^{n+2}\right) & =\frac{x_{n, 1}^{-}}{x_{n+1, n}^{-}} \Lambda^{-} .
\end{aligned}
$$

Of course, we can also express this in terms of the $\lambda_{i}$ by replacing $x_{i, j}^{+} \rightarrow\left\langle\lambda_{i} \lambda_{j}\right\rangle, x_{i, j}^{-} \rightarrow$ $\left\langle\tilde{\lambda}_{i} \tilde{\lambda}_{j}\right\rangle$.

\section{Constant monodromies}

The functional equations (3.28) are valid for the insertion of a generic operator. If it is an operator that can be described by a semiclassical string state, then we should put in (3.28) the monodromy of the corresponding state. In fact, for the so called "finite gap" states the monodromy can be computed in terms of an auxiliary hyper-elliptic Riemann surface. We will not derive the full formula for the area in these cases in this paper.

More generally, the equations (3.28) are valid even if a more complicated object is inserted in the $z \sim 0$ region. Of course, those more complicated objects would have more intricate monodromies, about which we know little. The full computation of the area will, almost certainly, require the computation of extra Y-functions beyond the ones we have introduced.

In his section, we concentrate on the simplest case. We consider monodromies inde-

pendent of the spectral parameter. We describe below some special cases, one of which corresponds to the insertion of operators with small conformal dimension. In this cases we will later derive the full formula for the area. 


\subsection{Recovering the $Y$-system for scattering amplitudes}

Let us consider the simplest possible monodromy

$$
\begin{aligned}
\hat{\Omega} & =\left(\begin{array}{ll}
1 & 0 \\
0 & 1
\end{array}\right) \\
\operatorname{Tr}[\hat{\Omega}] & =\operatorname{Tr}[\Omega]=2
\end{aligned}
$$

In this case, as we go around $z=0$ the solution goes to itself. All sections are single valued and $s_{i} \sim s_{i+n}$ and $x_{i+n}=x_{i}$. We see that $T_{n-1}=\left\langle s_{0} s_{n}\right\rangle=0$ and so $Y_{n-2}=0$. From the definition (3.30) we find $\bar{Y}=-1$. Thus, the Y-system is reduced to the on in 13

$$
Y_{s}^{+} Y_{s}^{-}=\left(1+Y_{s+1}\right)\left(1+Y_{s-1}\right), \quad s=1, \ldots, n-3
$$

4.2. Y-system for the form factors of operators with small dimension

The next case we would like to consider is

$$
\begin{aligned}
\hat{\Omega} & =\left(\begin{array}{ll}
1 & 0 \\
q & 1
\end{array}\right) \\
\operatorname{Tr}[\hat{\Omega}] & =\operatorname{Tr}[\Omega]=2 .
\end{aligned}
$$

Geometrically it corresponds to a solution which as we move around the operator insertion $z \rightarrow z e^{2 \pi i}$ transforms as $x\left(z e^{2 \pi i}\right)=x(z)+q, t\left(z e^{2 \pi i}\right)=t(z)$. It means that we consider solutions which are periodic in $x$ direction with period $q$. For the cusp positions it means $x_{i+n}^{ \pm}=x_{i}^{ \pm}+q$. As reviewed above, these solutions are the strong coupling dual of form factors of operators with small dimension. $q$ is momentum carried by the operator.

From the formula (3.32) it follows that (for this case $\Lambda=1$ )

$$
\begin{aligned}
& \bar{Y}(1)=\frac{q-x_{-\frac{n-1}{2},-\frac{n+1}{2}}^{+}}{x_{-\frac{n-1}{2},-\frac{n+1}{2}}^{+}}, \\
& \bar{Y}(i)=\frac{q-x_{-\frac{n-1}{2},-\frac{n+1}{2}}^{-}}{x_{-\frac{n-1}{2},-\frac{n+1}{2}}^{-}}
\end{aligned}
$$

Thus, we see that $\bar{Y}$ is a conformal invariant way of defining the period of the form factor solution. The Y-system in this case takes the form

$$
\begin{aligned}
Y_{s}^{+} Y_{s}^{-} & =\left(1+Y_{s+1}\right)\left(1+Y_{s-1}\right), \quad s=1, \ldots, n-3 \\
Y_{n-2}^{+} Y_{n-2}^{-} & =\left(1+Y_{n-3}\right)(1+\bar{Y})^{2} \\
\bar{Y}^{+} \bar{Y}^{-} & =1+Y_{n-2} .
\end{aligned}
$$


Since $\operatorname{Tr}[\Omega]=2$ but $\bar{Y} \neq-1$ the number of free parameters in the problem is $2(n-1)$.

This kinematical counting can be understood as follows. In this case we need $2(n+1)$ coordinates to fix the momenta of $2 n$ gluons. Restricting the monodromy to be a pure translation (rather than a more general conformal transformation) implies that we get $2(n-1)$ parameters. Alternatively, we can say we have $2 n$ light-like momenta, with $2 n$ parameters. Boosts and dilatations allow us to reduce this to $2(n-1)$ parameters.

\section{3. $Y$-system for $\mathrm{Z}_{\mathrm{m}}$ symmetric polygons}

As the last example we would like to consider the following monodromy

$$
\begin{aligned}
\hat{\Omega} & =\left(\begin{array}{cc}
\cos \left(\frac{\pi}{m}\right) & -\sin \left(\frac{\pi}{m}\right) \\
\sin \left(\frac{\pi}{m}\right) & \cos \left(\frac{\pi}{m}\right)
\end{array}\right) \\
\operatorname{Tr}[\hat{\Omega}] & =\operatorname{Tr}[\Omega]=2 \cos \left(\frac{\pi}{m}\right)
\end{aligned}
$$

We should emphasize that it is again $\zeta$ independent.

This corresponds to solutions where the global $A d S_{3}$ boundary coordinates obey $\phi\left(z e^{2 \pi i}\right)=\phi(z)+\frac{2 \pi}{m}, \tau\left(z e^{2 \pi i}\right)=\tau(z)$. Thus, we are dealing with $\mathrm{Z}_{\mathrm{m}}$ symmetric solutions. From the physical point of view they correspond to calculation of the scattering amplitudes with $\mathrm{Z}_{\mathrm{m}}$ symmetric kinematics. In the case when number of cusps is equal to $2 m$ these are regular polygons and they were considered in the literature before. More generally these are special subcases of Wilson loops with $2 \mathrm{~nm}$ cusps.

From the formula 3.32 it follows that

$$
\begin{aligned}
\delta \phi & =\phi_{-\frac{n-1}{2},-\frac{n+1}{2}} \\
\bar{Y}(1) & =\frac{\sin \left(\frac{\pi}{m}-\frac{\delta \phi^{+}}{2}\right)}{\sin \left(\frac{\delta \phi^{+}}{2}\right)}, \quad \phi^{+}=\phi+\tau \\
\bar{Y}(i) & =\frac{\sin \left(\frac{\pi}{m}-\frac{\delta \phi^{-}}{2}\right)}{\sin \left(\frac{\delta \phi^{-}}{2}\right)}, \quad \phi^{-}=\phi-\tau
\end{aligned}
$$

The Y-system takes the following form in this case

$$
\begin{aligned}
Y_{s}^{+} Y_{s}^{-} & =\left(1+Y_{s+1}\right)\left(1+Y_{s-1}\right), \quad s=1, \ldots, n-3 \\
Y_{n-2}^{+} Y_{n-2}^{-} & =\left(1+Y_{n-3}\right)\left(1+e^{\frac{\pi}{m} i} \bar{Y}\right)\left(1+e^{-\frac{\pi}{m} i} \bar{Y}\right) \\
\bar{Y}^{+} \bar{Y}^{-} & =1+Y_{n-2} .
\end{aligned}
$$


We can do one check of these equations immediately using the solution for the simplest case of regular polygons in $A d S_{3}$ which was considered in the literature [28]

$$
Y_{s}=\frac{\sin \left(\frac{\pi(s+2)}{n m}\right) \sin \left(\frac{\pi s}{n m}\right)}{\sin ^{2}\left(\frac{\pi}{n m}\right)}
$$

here the total number of gluons is $2 \mathrm{~nm}$ and the symmetry of the problem is $Z_{n m}$. However, we would like to consider this polygon as being $\mathrm{Z}_{\mathrm{m}}$ symmetric with $2 n$ cusps over one period and apply the Y-system written above to it.

From the (4.7) we get plugging $\delta \phi^{ \pm}=\frac{2 \pi}{n m}$ that

$$
\bar{Y}=\frac{\sin \left(\frac{\pi(n-1)}{n m}\right)}{\sin \left(\frac{\pi}{n m}\right)} .
$$

One can check that with these expressions the equations (4.8) are indeed satisfied.

These considerations allow us to make a connection to the family of solutions of modified Sinh-Gordon recently considered by Lukyanov and Zamolodchikov [29]. We relate

the parameter $l$ in 29] to our $m$ via $l=\frac{1}{2 m}-\frac{1}{2}$. Then the solutions described in [29] are special cases of the ones we consider here. When $m=1$ we come back to the problem of amplitudes. When $m=\infty$ we end up with the problem of form factor. For any integer $m$ we are dealing with the problem of calculation of the area of $\mathrm{Z}_{\mathrm{m}}$ symmetric polygons. This interplay is described in more detail in Appendix B. We do not know the physical interpretation for these solutions at general values of $l$.

\section{Y-system for the form factors}

In this section we focus our attention on the problem of minimal area surfaces in the massless BTZ black hole background, which corresponds to the problem of the form factor calculation. Firstly, we consider zig-zag solution to get the basic picture behind the problem. Then we turn to the analysis of analytical properties of Y-functions which allows us to write the integral form of the equations.

\subsection{Zig-zag solution}




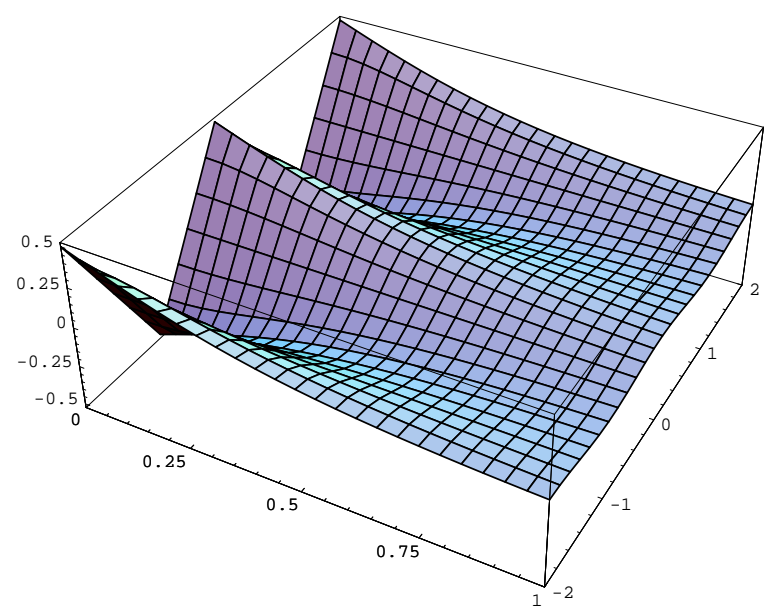

Fig. 6: The qualitative form of zig-zag solution which corresponds to the Sudakov form-factor. Here the Wilson line corresponds to momenta of gluons and is situated at $r=0$ operator is inserted at $r=\infty .\left\langle k_{2}|O(q)| k_{1}\right\rangle$ is related to the area of one period.

Here we analyze a zig-zag spacelike classical solutions in $A d S_{3}$ which corresponds to the following object at leading $\sqrt{\lambda}$ order:

$$
\begin{array}{r}
\left\langle k_{2}|O(q)| k_{1}\right\rangle \\
q=-\left(k_{1}+k_{2}\right)
\end{array}
$$

This is an operator decaying into two gluons. We are working in Poincare patch with the metric

$$
d s^{2}=\frac{d r^{2}-d t^{2}+d x^{2}}{r^{2}}
$$

We parameterize the worldsheet as $t(x, r)$ and introduce $\tilde{z}=r+i x$. For large $\tilde{z}$, or large $r$ the solution approaches the straight line Wilson loop solution which has $t=0$. In that case, the induced metric takes the form

$$
d s_{i n d}^{2}=\frac{d \tilde{z} d \overline{\tilde{z}}}{(\tilde{z}+\overline{\tilde{z}})^{2}}
$$

and the $p(\tilde{z})=0$. In order to implement the periodicity we can make the standard map from the strip to the unit disc

$$
z=e^{-\tilde{z}}
$$

then we have that near the origin induced metric takes the form

$$
d s_{i n d}^{2}=\frac{d z d \bar{z}}{z \bar{z} \log ^{2}(z \bar{z})}=e^{-2 \alpha} d z d \bar{z} .
$$


We will want to consider solutions which approach (5.5) when $z \rightarrow 0$. In particular, note that if we consider the polynomial $p(z)=\frac{1}{z}$ then in the $\tilde{z}$ coordinates it corresponds to $\tilde{p}(\tilde{z})=\left(\frac{\partial \tilde{z}}{\partial z}\right)^{-2} p(z)=e^{-\tilde{z}}$. For large $\tilde{z}$ the polynomial goes to 0 as we expect it for the class of solutions we consider. So we conclude that $p(z)=\frac{1}{z}$ is allowed in the class of solutions we want to consider. Notice that for higher poles we get $\tilde{p}(\tilde{z})$ that does not go to zero at large $\tilde{z}$, and we would change the qualitative form of the solution near $z \sim 0$.

It is natural then to expect that the form of the polynomial for $2 n$ gluons as the form

$$
p(z)=a_{n-2} z^{n-2}+a_{n-3} z^{n-3}+a_{n-4} z^{n-4}+\ldots+a_{0}+\frac{1}{z}
$$

where we used translations to set the pole at $z=0$ and rescaling to set the coefficient in front of the pole to one $\mathbf{\theta}$. We see that we have $n-1$ complex parameters $a_{i}$.

Let us summarize the picture that arises for the modified Sinh-Gordon in the table 5

\begin{tabular}{|c|c|c|}
\hline Case of $2 n$ gluons & Scattering Amp. & Operator Insertion \\
\hline Polynomial & $p(z)=a_{0}+a_{1} z+\ldots+z^{n-2}$ & $p(z)=\frac{1}{z}+\ldots+a_{n-2} z^{n-2}$ \\
\hline BC at $z=0$ & $\alpha$ regular & $\alpha \sim-\frac{1}{2} \log \left(z \bar{z} \log ^{2}(z \bar{z})\right)$ \\
\hline BC at $z=\infty$ & $\hat{\alpha} \rightarrow 0$ & $\hat{\alpha} \rightarrow 0$ \\
\hline
\end{tabular}

Table 1: Boundary conditions and polynomials in Modified Sinh-Gordon for operator insertion and scattering amplitudes.

We see that the kinematic information for $2 n$ gluons plus operator insertion is encoded in $2(n-1)$ real parameters of the polynomial which is what we expected from the Y-system counting. In the Appendix A we show that given boundary conditions for $\alpha,(5.5)$ and the general form of the polynomial (5.6) indeed lead to the off diagonal spectral independent monodromy which we used before.

Using this prescription and the techniques of [31,11] we can find the area of the one period for the zig-zag solution

$$
A_{\text {Sinh }}^{z z}=4 \int d^{2} w\left(e^{2 \hat{\alpha}}-1\right)=\frac{3 \pi}{4}
$$

4 Recall that the pole in the polynomial we consider is not what was called a "pole" in [17]. What was called a "pole" in their construction corresponds to the double pole in the polynomial we consider.

5 Solutions with given properties appeared in the past [30]. 
This is the answer for $n=1$, where we have no kinematic parameters. The simplest way to get this answer is to consider the limit $n \rightarrow \infty$ of the area for regular polygons and

notice that $A_{\text {Sinh }}^{z z}=\lim _{n \rightarrow \infty} \frac{A_{\text {Sinh }}^{r e g}(n)}{n}=\frac{3 \pi}{4}$, where we used the formula for the area of the regular polygon $A_{S i h n}(n)$ (formula (4.9) in [11]).

\subsection{Analytic properties of the Y-functions}

From the definition of $T$ 's it is clear that they are analytic functions of $\zeta$, for $\zeta \neq 0, \infty$. From this fact and the choice of normalization we made it also follows that $Y_{s}$ for $s \leq n-2$ are analytic away from $\zeta=0, \infty$ where they have an essential singularity. For $\bar{Y}$ it follows from $(3.27)$ and the fact that $B \neq 0$ for any $\zeta$.

For $\zeta \rightarrow 0, \infty$ we can analyze the flat section problem using a WKB analysis with $\zeta$ playing the role of $\hbar$. It was developed in previous work on the subject [17]. It was applied to the particular case of scattering amplitudes in [13]. Here the only new ingredient is the pole in the polynomial.

To be concrete let us consider the case when $\zeta \rightarrow 0$. Remember that we are considering the equation

$$
\left(d+A+\frac{\Phi_{z} d z}{\zeta}+\zeta \Phi_{\bar{z}} d \bar{z}\right) s=0
$$

For $\zeta \rightarrow 0$ it is convenient to make a complex gauge transformation which diagonalizes the Higgs field

$$
\Phi_{z}=\left(\begin{array}{cc}
\sqrt{p} & 0 \\
0 & -\sqrt{p}
\end{array}\right)
$$

Intuitively it is clear that the solutions of the flat section problem are dominated by the term $\frac{1}{\zeta} \Phi_{z}$ and have the approximate form $\left(\begin{array}{c}e^{-\frac{1}{\zeta} \int \sqrt{p} d z} \\ 0\end{array}\right)$ or $\left(\begin{array}{c}0 \\ e^{\frac{1}{\zeta}} \int^{0} \sqrt{p} d z\end{array}\right)$.

To make this statement more precise it is useful to introduce several notions. First of all it is natural to think about the problem as living on the Riemann surface $\Sigma$ given by

$$
x^{2}=p(z)
$$

On the complex plane $\mathcal{C}$ it corresponds to introducing branch cuts and working on two sheets. The differential $\lambda=\sqrt{p} d z$ that plays an important role is also living on the $\Sigma$ so on the complex plane and we should be careful about the sheet we are working on, namely $\lambda_{i}=(\sqrt{p},-\sqrt{p})$ where $i$ is the sheet label. 
Let us introduce the notion of WKB line, as a line where the exponent varies most rapidly

$$
\operatorname{Im}\left[\frac{\dot{z} \sqrt{p(z)}}{\zeta}\right]=0 .
$$

WKB lines live on the complex plane $\mathcal{C}$. Through each point on the complex plane only one WKB line is going through. The direction of the WKB line shows the direction towards which the solution increases.

Then more precise statement is that small solutions in the limit $\zeta \rightarrow 0$ take the form

$$
\begin{aligned}
& s \sim c(z)\left(\begin{array}{c}
e^{-\frac{1}{\zeta} \int \lambda_{1}} \\
0
\end{array}\right) \\
& s \sim c(z)\left(\begin{array}{c}
0 \\
e^{-\frac{1}{\zeta} \int \lambda_{2}}
\end{array}\right) .
\end{aligned}
$$

where $c(z)$ does not depend on $\zeta$. Thus, $s_{i}$ lives on the $(i+1)$-th sheet $\bmod (2)$.

An important observation is that if we have a WKB line which connects Stokes sectors $i$ and $j$, we can use it to evaluate $\left\langle s_{i} s_{j}\right\rangle$. Using this fact we can reliably find the asymptotic behavior of the Y-functions [17].

\subsection{Evaluating $Y^{\prime}$ 's}

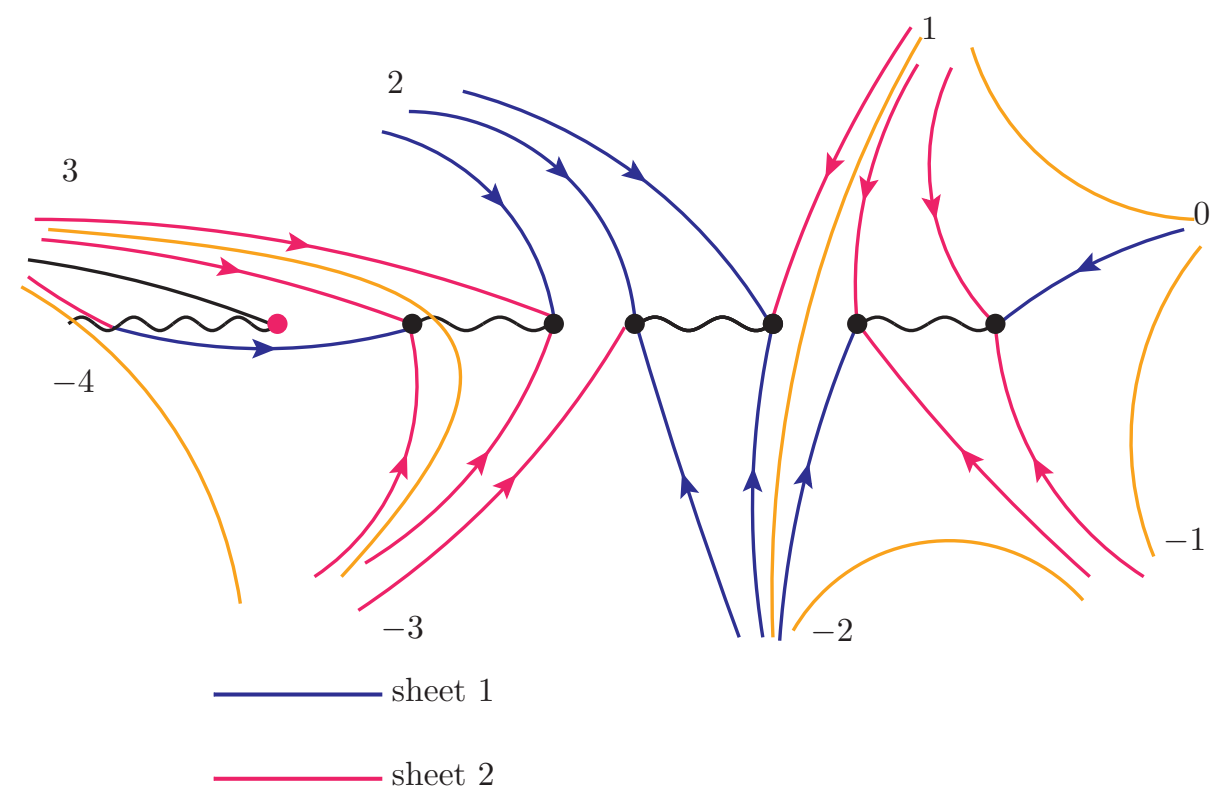

Fig. 7: Here we present the approximate form of the flow for $\zeta=e^{i \frac{\pi}{4}}$ and $n=7$. Black dots represent zeroes of $p(z)$ while the red one is the pole. The numbers label different Stokes sectors. Blue and red lines which ends on zeroes separate different groups of WKB lines. The orange lines show the cycle along which we evaluate $Y_{1}$ (on the right) and $\bar{Y}$ (on the left). 
For convenience we choose $p(z)=\frac{\prod\left(\frac{z}{z_{i}}-1\right)}{z}$ with all zeroes being positive and located on real axis. The only difference with the current situation compared to the amplitude case is the different flow pattern around the pole, which we located to the left, see fig. 7 . There are three lines ending at each zero and one line ending at the pole. The lines that end at the zeros or poles separate different flow patterns of WKB lines (or different basins of attraction).

The asymptotic behavior of the Y-functions at $\zeta=0, \infty$ is again given by contour integrals around certain cycles. Compared to the amplitude case, for the same value of $n$, added an extra pole and an extra zero to the polynomial. Thus, two more cycles appear. This is another manifestation of appearance of 2 additional Y-functions in the Y-system for the form factor compare to the case of amplitudes.

The only new feature here is the evaluation of the $\bar{Y}$ function. Form the definition, (3.30) and figure fig. 5, it is clear that we can evaluate it using the contour indicated in figure fig. 7 .

The story happens to be completely identical to the amplitudes one. Here we just present the results. Asymptotic behavior of Y-functions is given by (introducing $\zeta=e^{\theta}$ )

$$
\begin{aligned}
\log Y_{s} & =-m_{s} \cosh \theta+\ldots, \quad s=1, \ldots, n-2 \\
\log \bar{Y} & =-\bar{m} \cosh \theta+\ldots,
\end{aligned}
$$

where

$$
\begin{aligned}
m_{2 k} & =2 \oint_{\gamma_{2 k}} \lambda \\
m_{2 k+1} & =-2 i \oint_{\gamma_{2 k+1}} \lambda \\
\bar{m} & =2 \oint_{\bar{\gamma}} \lambda
\end{aligned}
$$

these formulas are valid because with our choice of the polynomial all masses are real and positive. Here one should be as usually careful with the contour orientation and the sheet where the differential is considered.

If we slightly move the zeroes $p(z)$ away from the real axis the $m_{s}$ in (5.14) become complex. The asymptotic behavior of Y-functions is $\log Y_{s} \sim-\frac{m_{s}}{2} e^{-\theta}$ for $\theta \rightarrow+\infty$ and $\log Y_{s} \sim-\frac{m_{s}^{*}}{2} e^{\theta}$ for $\theta \rightarrow-\infty$. 


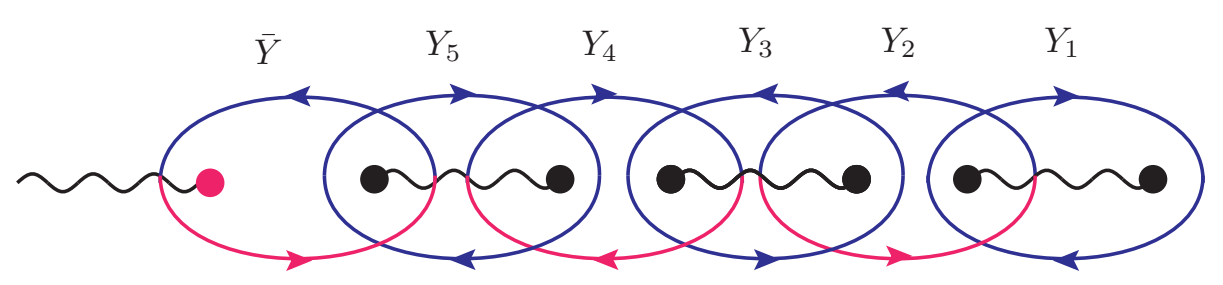

Fig. 8: The cycles which we need to integrate $\lambda$ along to determine the asymptotic behavior of all Y-functions (case $n=7$ ). For the general odd $n$ intersection form is $\theta^{s r}=(-1)^{s+1}\left(\delta_{s+1, r}+\delta_{s-1, r}\right)$.

\subsection{Integral form of the equations}

Functional equations supplemented with the asymptotic behavior of the Y-functions specifies the solution of Y-system uniquely. It is convenient to rewrite the equations in an integral form which is completely analogous to the case of amplitudes. If we introduce the kernel $K(\theta)=\frac{1}{2 \pi \cosh \theta}$ and the operation star being the convolution $K \star f=\int_{-\infty}^{\infty} d \theta^{\prime} K(\theta-$ $\left.\theta^{\prime}\right) f\left(\theta^{\prime}\right)$ then the system of integral equations for the Y-functions as functions of the spectral parameter takes the form

$$
\begin{aligned}
\log Y_{s} & =-m_{s} \cosh \theta+K \star \log \left(1+Y_{s+1}\right)+K \star \log \left(1+Y_{s-1}\right), \quad s=1, \ldots, n-3 \\
\log Y_{n-2} & =-m_{n-2} \cosh \theta+K \star \log \left(1+Y_{n-3}\right)+2 K \star \log (1+\bar{Y}) \\
\log \bar{Y} & =-\bar{m} \cosh \theta+K \star \log \left(1+Y_{n-2}\right)
\end{aligned}
$$

where the fact that masses are real was used. The form of the integral equations in the general case of complex masses is very similar and it can be found in Appendix D. If the phases of the $m_{s}$ become very large, then one needs to perform wall crossing transformations.

We can also consider the case when the trace of the monodromy is $\operatorname{Tr}[\tilde{\Omega}]=2 \cosh \left(\frac{\mu}{\zeta}+\right.$ $\bar{\mu} \zeta)$. This could arise if we replace the single pole at $z=0$ by a double pole and a closely located zero, $1 / z \rightarrow \frac{\mu^{2}}{4 \pi^{2} \epsilon} \frac{z-\epsilon}{z^{2}}$ and also claim that $\hat{\alpha} \rightarrow 0$ near the origin 6 . In this situation, integral equations continue to be the same as long as $2|\mu|<|\bar{m}|$. When this condition is not obeyed, it might be necessary to do wall crossing. Note that in this case we can define new $Y$ functions $\bar{Y}_{\hat{1}}=e^{\mp\left(\frac{\mu}{\zeta}+\bar{\mu} \zeta\right)} \bar{Y}$. We can then write the two final equations of the $\mathrm{Y}$ systems as

$$
\begin{aligned}
Y_{n-2}^{+} Y_{n-2}^{-} & =\left(1+Y_{n-3}\right)\left(1+\bar{Y}_{\hat{+}}\right)\left(1+\bar{Y}_{\hat{\lrcorner}}\right) \\
\bar{Y}_{\hat{+}}^{+} \bar{Y}_{\hat{+}}^{-} & =\left(1+Y_{n-2}\right), \quad \bar{Y}_{\dot{\iota}}^{+} \bar{Y}_{\grave{\iota}}^{-}=\left(1+Y_{n-2}\right)
\end{aligned}
$$

The masses then are $\bar{m}_{\hat{1}}= \pm 2 \mu+\bar{m}$.

6 These boundary conditions are natural from the [17] point of view. 


\section{Area in the case of form factors}

In this section we present the formula for the area first in the form which contain mass parameters and then in the form which depends only on cross ratios. We consider the case that the monodromy is constant and off diagonal, which corresponds to the computation of form factors of operators with small conformal dimensions.

\subsection{General formula}

We start from the following divergent integralt to calculate

$$
A=2 \int d^{2} z \operatorname{Tr}\left[\Phi_{z} \Phi_{\bar{z}}\right]
$$

one can rewrite it as follows (for $n$ odd)

$$
\begin{aligned}
A & =A_{\text {reg }}+A_{\text {periods }}+A_{\text {cutoff }} \\
A_{\text {reg }} & =A_{\text {Sinh }}=4 \int d^{2} w\left(e^{2 \hat{\alpha}}-1\right)=\int d^{2} z\left(2 \operatorname{Tr}\left[\Phi_{z} \Phi_{\bar{z}}\right]-4(p \bar{p})^{1 / 2}\right) \\
A_{\text {periods }} & =4 \int d^{2} z(p \bar{p})^{1 / 2}-4 \int_{\Sigma_{0}} d^{2} w=4 \int_{\Sigma} d^{2} w-4 \int_{\Sigma_{0}} d^{2} w \\
A_{\text {cutoff }} & =4 \int_{\Sigma_{0}, z_{\text {AdS }}>\epsilon} d^{2} w .
\end{aligned}
$$

The simplest part is $A_{\text {cutoff }}=A_{\text {div }}+A_{B D S-l i k e}$

$$
\begin{aligned}
A_{d i v} & =\sum_{i=1}^{2 n} \frac{1}{8}\left(\log \epsilon^{2} s_{i, i+2}\right)^{2} \\
A_{B D S-l i k e} & =l_{i}^{+} M_{i j} l_{j}^{-} \\
l_{i}^{+} & =\log \left(x_{i+1}^{+}-x_{i}^{+}\right), \quad l_{i}^{-}=\log \left(x_{i+1}^{-}-x_{i}^{-}\right)
\end{aligned}
$$

where again $l_{i+n}^{ \pm}=l_{i}^{ \pm}$and $M_{i j}$ can be read off from (5.8) in [12]. The proof that it takes the same form as in the case of amplitudes is given in the Appendix C. Qualitatively it can be understood from the fact that both terms depend only on the difference of coordinates between two consecutive cusps so at most the part which involves the difference between the $n$-th and first cusp can change due to the presence of the monodromy. However, in the case of periodic polygons even this does not happen.

7 To be more precise the area $4 \int d^{2} z e^{2 \alpha}=2 \int d^{2} z \operatorname{Tr}\left[\Phi_{z} \Phi_{\bar{z}}\right]+2 \int d^{2} z \partial \bar{\partial} \alpha$. For the case of form factor $2 \int d^{2} z \partial \bar{\partial} \alpha=\frac{\pi n}{2}$. A term proportional to $n$ can be absorbed by a redefinition of the regularization procedure of the cusps. 


\subsection{Area as the free energy}

The non-trivial finite piece of the area is

$$
A_{\text {Sinh }}^{f f}=A_{\text {free }}^{f f}+C_{z z}+(n-1) C_{6}, \quad C_{z z}=\frac{3 \pi}{4}, \quad C_{6}=\frac{7 \pi}{12}
$$

here $C_{z z}$ is the area of the zig-zag solution (5.7) and $C_{6}$ is the area of the regular hexagon. One can get this formula as follows. When all zeroes and the pole are far from each other the free energy for the form factor Y-system goes to zero. Since $\hat{\alpha}$ is massive field we get isolated contributions from the zeroes and the pole. Each pole correspond to the regular hexagon while the pole to the area of the zig-zag solution.

For $A_{\text {periods }}$ one gets the same expression as in the case of amplitudes [13], namely

$$
A_{\text {periods }}=-i w_{s, s^{\prime}} Z^{s} Z^{s^{\prime}}
$$

where $w_{s, s^{\prime}}$ is the inverse of the intersection form and $Z^{2 k}=-\frac{m_{2 k}}{2}, Z^{2 k+1}=-i \frac{m_{2 k+1}}{2}$, $Z^{n-1}=-\frac{\bar{m}}{2}$.

$A_{\text {free }}$ in the formula above is given by the free energy of the TBA system

$$
\begin{aligned}
A_{\text {free }}^{f f} & =\sum_{s} \int \frac{d \theta}{2 \pi}\left|m_{s}\right| \cosh \theta \log \left(1+\tilde{Y}_{s}\right)+2 \int \frac{d \theta}{2 \pi}|\bar{m}| \cosh \theta \log (1+\tilde{\bar{Y}}) \\
m_{s} & =\left|m_{s}\right| e^{i \phi_{s}} \quad \bar{m}=|\bar{m}| e^{i \bar{\phi}} \\
\tilde{Y}_{s}(\theta) & =Y_{s}\left(\theta+i \phi_{s}\right), \quad \tilde{\bar{Y}}=\bar{Y}(\theta+i \bar{\phi})
\end{aligned}
$$

Notice the appearance of an extra factor of 2 in front of term containing $\bar{Y}$. This factor plays an important role in all calculations below. One sees that this formula contains $2(n-1)$ parameters as it should. The whole kinematics is encoded in the mass parameters. We can get rid of the masses and write the area purely in terms of the physical cross ratios which is described below.

\subsection{Area as the critical value of Yang-Yang functional}

In [8] the expression for the area purely in terms of cross ratios was obtained. The same can be done in the case of form factor with minor changes in the discrete data and the number of Y-functions.

It is convenient to introduce new variables

$$
\begin{aligned}
X_{2 k}(\theta) & =Y_{2 k}(\theta), \quad X_{2 k+1}(\theta)=Y_{2 k+1}\left(\theta-i \frac{\pi}{2}\right) \\
X_{n-1}(\theta) & =\bar{Y}(\theta)
\end{aligned}
$$


for which $X_{s}(\zeta=1)=\chi_{s}^{+}$and $X_{s}(\zeta=i)=\chi_{s}^{-}$where $\chi_{s}^{ \pm}$are physical cross ratios that define kinematics of the process.

In terms of these variables we can rewrite the Y-system in the form given in equation D.2 of [8] with the discrete data $\langle s, s+1\rangle=-\langle s+1, s\rangle=(-1)^{s+1}$ and

$$
\begin{aligned}
\Omega(s) & =1, \quad s=1, . ., n-2 \\
\Omega(n-1) & =2 .
\end{aligned}
$$

We can use the formulas given in [8] for the area $A_{\text {periods }}^{f f}+A_{\text {free }}^{f f}=A_{0}^{f f}+Y Y_{c r}$ with

$$
\begin{aligned}
A_{0}^{f f}= & -\frac{1}{2} \sum_{s, s^{\prime}=1}^{n-1} w_{s, s^{\prime}} \log \chi_{s}^{+} \log \chi_{s^{\prime}}^{-} \\
Y Y_{c r}= & \frac{1}{\pi} \sum_{s=1}^{n-1} \Omega(s) \int_{l_{s}} \frac{d \theta}{\sinh ^{2} \theta} \operatorname{Li}_{2}\left(-X_{s}(\theta)\right)+ \\
& +\frac{1}{4 \pi^{2} i} \sum_{s, s^{\prime}=1}^{n-1} \Omega(s) \Omega\left(s^{\prime}\right)\left\langle s, s^{\prime}\right\rangle \int_{l_{s^{\prime}}} \frac{d \theta^{\prime}}{\sinh 2 \theta^{\prime}} \int_{l_{s}} \frac{d \theta}{\sinh 2 \theta} \\
& \frac{1}{\sinh \left(\theta^{\prime}-\theta\right)} \log \left(1+X_{s^{\prime}}\left(\theta^{\prime}\right)\right) \log \left(1+X_{s}(\theta)\right) .
\end{aligned}
$$

The integral equations take the form in equation D.3 of [8]. To fix the contours of integration we go to the region of parameter space where all cross ratios are very small or very large. In this region the simple relation between masses and cross ratios exist so we can fix the contours of integration. After it being careful about contribution from the poles in the kernels we can continue them to the region of arbitrary cross ratios.

\section{Exact solutions}

In this section we consider several simple cases when the solution of the Y-system is known and the area can be found exactly. The simplest case is when Y-functions are independent of the spectral parameter. These arise when all masses go to zero. They correspond to the high temperature limit of the TBA. Geometrically they correspond to a polygon with maximal symmetry. In addition, an exact solution can be found for the form factors case for $n=2$. This solution is very similar to that of the octagonal Wilson loop [11]. 


\subsection{High temperature limit of the form factor Y-system}

Here we consider solutions of the form factor Y-system which are independent of the spectral parameter. Geometrically the regular form factor corresponds to the same zig-zag solution fig. 6 , but with a different choice for the number of cusps per period.

In the case of amplitudes the answer is known to be

$$
\begin{aligned}
A_{\text {Sinh }}^{s a}(n) & =A_{\text {free }}^{s a}(n)+(n-2) C_{6}=\frac{\pi}{4 n}\left(3 n^{2}-8 n+4\right) \\
A_{\text {free }}^{s a}(n) & =\frac{\pi}{6} \frac{(n-3)(n-2)}{n}
\end{aligned}
$$

where $2 n$ is the total number of gluons in the problem.

For the form factor we have

$$
A_{\text {Sinh }}^{f f}(n)=A_{\text {free }}^{f f}(n)+C_{z z}+(n-1) C_{6}
$$

where $2 n$ is again the total number of gluons. From the definition of the regular form factor we see that $A_{\text {Sinh }}^{f f}(n)=n C_{z z}$. Thus, we get the expression for the free energy

$$
\begin{aligned}
n C_{z z} & =A_{\text {free }}^{f f}(n)+C_{z z}+(n-1) C_{6} \\
A_{\text {free }}^{f f}(n) & =(n-1)\left(C_{z z}-C_{6}\right)=\frac{\pi}{6}(n-1) .
\end{aligned}
$$

The first check of this formula can be done for the cases when we have only one or two non-zero Y-functions in the form factor problem. One can notice looking at corresponding Y-systems (4.2) and (4.5) that they are completely equivalent in these cases in high temperature limit and the following equalities should hold

$$
\begin{aligned}
& A_{\text {free }}^{f f}(2)=2 A_{\text {free }}^{s a}(4), \\
& A_{\text {free }}^{f f}(3)=A_{\text {free }}^{s a}(6) .
\end{aligned}
$$

Using the formulas (7.1) and (7.3) one can check that this is indeed true.

More non-trivial check is to reproduce the result for the free energy of regular form factor (7.3) using the Y-system. If we take the zig-zag solution and we choose the period to contain $2 n$ cusps then the solution of (4.5) is

$$
\begin{aligned}
Y_{s} & =s(s+2) \\
\bar{Y} & =n-1
\end{aligned}
$$

8 Using different language it can be understood at the level of Dynkin diagrams as $D_{2}=2 A_{1}$ and $D_{3}=A_{3}$ relations where the form factor Y-system corresponds to $D_{n}$ series and the scattering amplitudes one to $A_{n}$ series [32]. 
one can get this solution in two ways: either from geometrical definition of the Y-functions or by taking large $n$ limit of the regular polygon solution for $A d S_{3}$ (4.9), (4.10).

The formula for the free energy in this case takes the form [33]:

$$
\begin{aligned}
A_{f r e e}^{f f}(n) & =-\frac{1}{2 \pi} \sum_{s=1}^{n-2}\left(\log \left(Y_{s}\right) \log \left(1+Y_{s}\right)+2 L i_{2}\left(-Y_{s}\right)\right) \\
& -\frac{1}{\pi}\left(\log (\bar{Y}) \log (1+\bar{Y})+2 L i_{2}(-\bar{Y})\right)
\end{aligned}
$$

inserting values for Y's (7.5) one can check that this sum is indeed equal to $\frac{\pi}{6}(n-1)$.

Another check can be done by viewing the form factor as an infinite number of gluons limit of the amplitude

$$
A_{\text {Sinh }}^{f f}(n)=\lim _{m \rightarrow \infty} \frac{A_{\text {Sinh }}^{s a}(n m)}{m}=n C_{z z}
$$

which also holds.

\subsection{High temperature limit of the $\mathrm{Z}_{\mathrm{m}}$ symmetric $Y$-system}

Using the known results for the area of regular polygons we can make a consistency check for the $\mathrm{Z}_{\mathrm{m}}$ symmetric $\mathrm{Y}$-system.

Namely let us consider a regular polygon with $2 m n$ cusps and view it as a special case of a $\mathrm{Z}_{\mathrm{m}}$ symmetric one. By the construction

$$
\begin{aligned}
m A_{\operatorname{Sinh}}^{Z_{m}}(n) & =A_{\text {Sinh }}^{s a}(n m) \\
A_{\text {Sinh }}^{s a}(n m) & =A_{\text {free }}^{s a}(n m)+(n m-2) C_{6} \\
A_{\text {Sinh }}^{Z_{m}}(n) & =A_{\text {free }}^{Z_{m}}(n)+C_{p}(m)+(n-1) C_{6}
\end{aligned}
$$

where we again consider the limit when all zeroes and pole are far away from each other to get the second and the third formulas. We introduced the $C_{p}(m)$ for the area of the isolated pole. It should appear as the area for the solution of modified Sinh-Gordon with $p(z)=\frac{1}{z}$ and a boundary condition for $\alpha$ given in Appendix B.

By considering (7.8) for $n=1$ we get

$$
C_{p}(m)=\frac{A_{\text {Sinh }}^{s a}(m)}{m}=\frac{A_{\text {free }}^{s a}}{m}+\frac{m-2}{m} C_{6}=\frac{\pi(m-2)(3 m-2)}{4 m^{2}} .
$$

Putting all together we get

$$
A_{\text {free }}^{Z_{m}}(n)=\frac{A_{\text {free }}^{s a}(n m)-A_{\text {free }}^{s a}(m)}{m}=\frac{\pi}{6}(n-1)\left(1-\frac{6}{m^{2} n}\right) .
$$


Again it is interesting to reproduce this result directly from the Y-system. In the case of $\mathrm{Z}_{\mathrm{m}}$ symmetric $\mathrm{Y}$-system (4.8) the free energy takes the form

$$
\begin{aligned}
A_{\text {free }}^{Z_{m}}(n) & =-\frac{1}{2 \pi} \sum_{s=1}^{n-2}\left(\log \left(Y_{s}\right) \log \left(1+Y_{s}\right)+2 L i_{2}\left(-Y_{s}\right)\right) \\
& -\frac{1}{2 \pi}\left(\log (\bar{Y}) \log \left(1+e^{i \frac{\pi}{m}} \bar{Y}\right)+2 L i_{2}\left(-e^{i \frac{\pi}{m}} \bar{Y}\right)\right) \\
& -\frac{1}{2 \pi}\left(\log (\bar{Y}) \log \left(1+e^{-i \frac{\pi}{m}} \bar{Y}\right)+2 L i_{2}\left(-e^{-i \frac{\pi}{m}} \bar{Y}\right)\right) .
\end{aligned}
$$

Plugging the solution (4.9), (4.10) into (7.11) we reproduce (7.10). Another point is that we expect from the form factor as an infinite number of gluon limit the following equations to be satisfied

$$
\begin{aligned}
A_{\text {free }}^{f f}(n) & =\lim _{m \rightarrow \infty} A_{\text {free }}^{Z_{m}}(n) \\
C_{z z} & =\lim _{m \rightarrow \infty} C_{p}(m) .
\end{aligned}
$$

One can easily check that this is indeed true.

\subsection{Exact solution for the 4-cusp form factor}

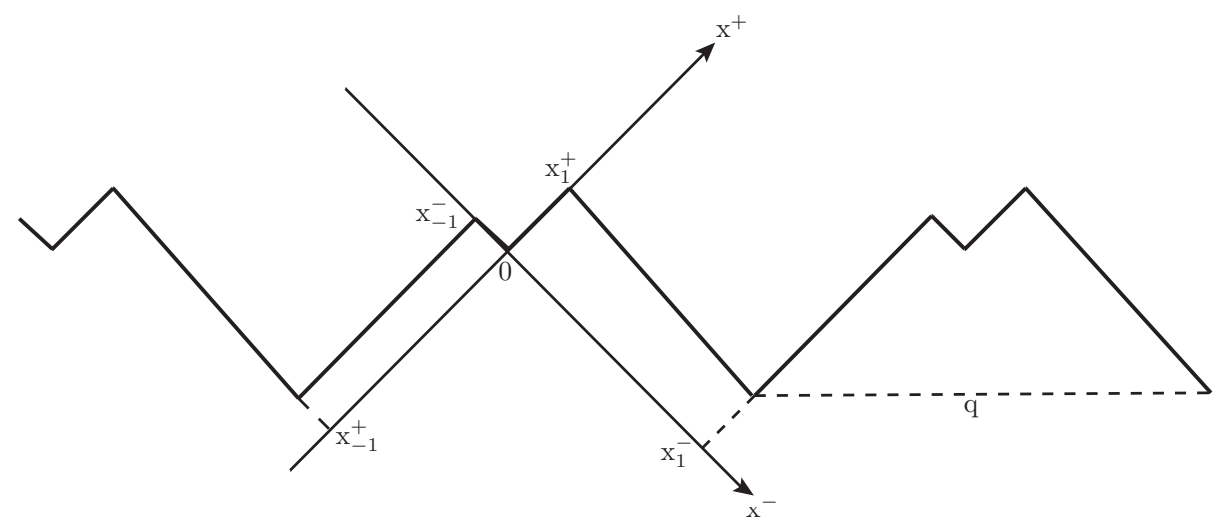

Fig. 9: We consider an operator going into states with 4 gluons which at strong coupling is given by the surface with boundary condition shown in the picture. The zeroth cusp chosen to be at the origin. The momentum of the operator is $q$. The picture corresponds to the case when $\chi^{+} \ll 1$ and $\chi^{-} \gg 1$.

Here we give the exact solution for the area in the case of 4-cusp form factor depicted in fig. 9. Here $n=2$ and the Y-system is just $\bar{Y}^{+} \bar{Y}^{-}=1$, with the solution $\bar{Y}=e^{Z / \zeta+\bar{Z} \zeta}$. 
We denote the only cross ratio in the problem by $\chi$ with the definition

$$
\begin{gathered}
\bar{Y}(\zeta=1)=\chi^{+}=\frac{x_{1,0}^{+}}{x_{0,-1}^{+}} \\
\bar{Y}(\zeta=i)=\chi^{-}=\frac{x_{1,0}^{-}}{x_{0,-1}^{-}}
\end{gathered}
$$

Due to the fact that $n$ is even in this case we cannot directly apply formulas we have written above (6.2), (6.3). However, we can derive the correct result by viewing this case as the the double soft limit from the $n=3$ form factor problem. This is explained in detail in Appendix E. The case of 4-cusp form factor is analogous to the octagon up to the several important coefficients and signs. Also here we do not subtract from the area $A_{B D S}$ but take the limit directly at the level of $A_{B D S-l i k e}$.

With given definitions the answer for the area is

$$
\begin{aligned}
A & =A_{\text {div }}+\tilde{A}_{B D S-l i k e}+R \\
\tilde{A}_{B D S-l i k e} & =\frac{1}{4}\left(\log \chi^{-} \log \chi^{+}+\log \chi^{-} \log \left(1+\chi^{+}\right)^{2}-\log \chi^{+} \log \left(1+\chi^{-}\right)^{2}\right) \\
R & =\frac{4 \pi}{3}+2 I \\
I & =\int_{-\infty}^{\infty} d t \frac{|m| \sinh t}{2 \pi \tanh (2 t+2 i \phi)} \log \left(1+e^{-2|m| \cosh t}\right), \quad \phi \in\left(0, \frac{\pi}{2}\right)
\end{aligned}
$$

where

$$
\log \chi^{+}=-|m| \sin \phi, \quad \log \chi^{-}=|m| \cos \phi
$$

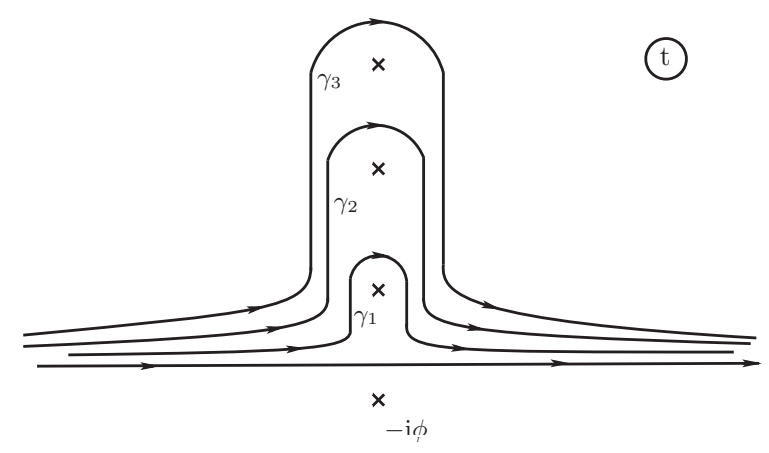

Fig. 10: Here the complex $t$ plane is presented with the poles located at $-i \phi+i \frac{\pi n}{2}$. We show contours $\gamma_{i}$ which enter in the definition of $I_{\text {periodic }}$. 
Written in this form it is not obvious that the answer is invariant under cyclic permutations or spacetime parity. It is due to the fact that as we increase $\phi$ poles can cross the real line which we are integrating over. The deformation of the contour can be rewritten as the sum of real line integral plus the integral encircling the pole [11]. To make this symmetries manifest it is useful to define the function $I_{\text {periodic }}=\frac{1}{4}\left(I+I_{\gamma_{1}}+I_{\gamma_{2}}+I_{\gamma_{3}}\right)$ where $I$ is the integral over the real line and $\gamma_{i}$ are contours shown in fig. 10 with the same integrand as in (7.14). Then $I_{\text {periodic }}\left(\phi+\frac{\pi}{2}\right)=I_{\text {periodic }}(\phi)$ and one can show that

$$
2 I=2 I_{\text {periodic }}-\tilde{A}_{B D S-l i k e}
$$

and the answer for the area can be written as

$$
A-A_{d i v}=\frac{4 \pi}{3}+2 I_{\text {periodic }}(|m|, \phi) .
$$

Written in this form the area exhibits the explicit spacetime parity and cyclicity symmetries. In the $|m| \rightarrow 0$ limit we have $A-A_{d i v}=\frac{4 \pi}{3}+2 \frac{\pi}{12}=\frac{3 \pi}{2}$ which is the correct answer for the two copies of zig-zag.

Analogously we can get the critical Yang-Yang functional of the $Z_{m}$ symmetric polygon with $4 m$ number of gluons

$$
\begin{aligned}
Y Y^{Z_{m}}(2) & =\int_{-\infty}^{\infty} d t \frac{|m| \sinh t}{2 \pi \tanh (2 t+2 i \phi)} \log \left(1+e^{i \frac{\pi}{m}} e^{-2|m| \cosh t}\right)\left(1+e^{-i \frac{\pi}{m}} e^{-2|m| \cosh t}\right), \\
\phi & \in\left(0, \frac{\pi}{2}\right)
\end{aligned}
$$

where

$$
\log \chi^{+}=-|m| \sin \phi, \quad \log \chi^{-}=|m| \cos \phi
$$

and

$$
\chi^{+}=\frac{\sin \frac{\phi_{1,0}^{+}}{2}}{\sin \frac{\phi_{0,-1}^{+}}{2}}, \quad \chi^{-}=\frac{\sin \frac{\phi_{1,0}^{-}}{2}}{\sin \frac{\phi_{0,-1}^{-}}{2}} .
$$

One can show that at $m=2$ it correctly reproduces the answer for the octagon while in the limit $m \rightarrow \infty$ it goes to the solution for the four cusp form factor. 


\section{Conclusions}

In this paper we have considered the problem of calculating form factors at strong coupling, limiting ourselves to $R^{1,1}$ kinematics.

These are given by the area of minimal surfaces in $A d S$ space which end on a periodic sequence of null segments at the boundary of $A d S$ space. The shape of the sequence is fixed by the gluon momenta. The operator momentum defines the period of the sequence.

The problem can be reformulated in terms of a flat section problem for the spectral parameter dependent flat connection. The insertion of the operator creates a non-trivial monodromy on the worldsheet which specifies the behavior of the connection near the insertion point. This monodromy characterizes the operator. Without an operator insertion (with a trivial monodromy) this problem was recently solved using the integrability of classical strings in $A d S$ [13]. In that case, one needs to solve a set of functional equations for the cross ratios as functions of the spectral parameter with given boundary condition at $\theta \rightarrow \pm \infty$ - the Y-system. Here we extended the previous analysis and derived a set of functional equations for the insertion of a general operator (3.28). In fact, these functional equations should be valid for general operators, even those dual to semiclassical string states, or the ones described by "finite gap" solutions [18,19]. They should even be valid in cases where we have any other structure near $z=0$, such as another Wilson loop, though in such cases we would probably need more Y-functions to fully specify the system. In other words, the equations (3.28) are a local property of the irregular singularity at infinity and the holonomy around it.

We have then concentrated on a couple of examples when the monodromy does not depend on the spectral parameter. One corresponds to the case of form factors of operators with conformal dimensions small compared to $\sqrt{\lambda}$. The monodromy can be found explicitly (4.3). Supplementing the functional equations (4.5) with boundary conditions using a WKB analysis, we rewrote them in the form of integral equations of the TBA form (5.15). The area is then given by the free energy of the TBA system or critical value of Yang-Yang functional. (6.6), (6.9).

It should be noted that, at leading order, the answer is insensitive to the particular form of the operator. This has a physical explanation: at strong coupling, the production of finite number of quanta is equally strongly suppressed for all operators [22,23]. The dependence on the precise operator, as well as the dependence on the gluon polarizations should reappear at one loop in the $1 / \sqrt{\lambda}$ expansion. 
First the analysis was done for the case when we have an operator and $2 n$ gluons with $n$ being odd. The case of even $n$ 's can be then obtained as a simple double soft limit which we explained both for the case of amplitudes and form factor (see Appendix E).

Using this knowledge we solved exactly the problem for an operator creating 4 gluons. The area is given by (7.17). Another easily tractable case is the so called high temperature limit. In this case the Y-functions do not depend on the spectral parameter and the solution of the Y-system can also be found. We used it as a non-trivial check of our formulas.

This analysis can be extended in a few other directions. The most obvious one is to generalize it to the case of $A d S_{5}$ or full $R^{1,3}$ kinematics. Another direction is to consider the insertion of more general operators which are given by classical string solutions at strong coupling and for which the anomalous dimension at leading order is non-zero. One famous example of such a solution is GKP string [34]. In this case we will have the functional equations derived in (3.28) where $\operatorname{Tr}[\tilde{\Omega}]$ should be the one corresponding to the operator. What remains to be done is the derivation of the expression for the area. This could require the introduction of extra functions. Another direction is to extend the present analysis to the case of an insertion of several operators which can then be related to the problem of the calculation of correlation functions in $\mathcal{N}=4 \mathrm{SYM}$ at strong coupling. One more question is whether there is a form factor/Wilson line analogue of the scattering amplitudes/Wilson loop duality at weak coupling.

\section{Acknowledgments}

We thank Benjamin Basso for collaboration at the beginning of the project. We also thank Luis F. Alday, Davide Gaiotto, Pedro Vieira and Amit Sever, for very useful comments and suggestions, as well as for collaboration in closely related issues.

\section{Appendix A. Derivation of the monodromy}

Here we present the derivation for the monodromy in the case of an operator insertion. We use the explicit solution of the section problem in the vicinity of operator insertion. This is given by the following equations

$$
\begin{aligned}
& \partial_{z} \psi+B_{z}(\zeta) \psi=0 \\
& \partial_{\bar{z}} \psi+B_{\bar{z}}(\zeta) \psi=0
\end{aligned}
$$


where

$$
B_{z}=\left(\begin{array}{cc}
\frac{1}{2} \partial_{z} \alpha & -\frac{1}{\zeta} e^{\alpha} \\
-\frac{1}{\zeta} e^{-\alpha} p(z) & -\frac{1}{2} \partial_{z} \alpha
\end{array}\right), \quad B_{\bar{z}}=\left(\begin{array}{cc}
-\frac{1}{2} \partial_{\bar{z}} \alpha & -\zeta e^{-\alpha} \bar{p}(\bar{z}) \\
-\zeta e^{\alpha} & \frac{1}{2} \partial_{\bar{z}} \alpha
\end{array}\right)
$$

are components of the flat connection which appears in the reduced formalism. The connection is defined in terms of a solution of modified Sinh-Gordon $\alpha(z, \bar{z})$ and the polynomial $p(z)$ that controls the kinematics of the process. It is convenient to make a gauge transformation

$$
\begin{aligned}
\partial_{z}+\hat{B}_{z} & =g\left[\partial_{z}+B_{z}\right] g^{-1} \\
\partial_{\bar{z}}+\hat{B}_{\bar{z}} & =g\left[\partial_{\bar{z}}+B_{\bar{z}}\right] g^{-1} \\
\hat{\psi} & =g \psi
\end{aligned}
$$

with

$$
g=\left(\begin{array}{cc}
\left(\frac{z}{\bar{z}}\right)^{-1 / 4} & 0 \\
0 & \left(\frac{z}{\bar{z}}\right)^{1 / 4}
\end{array}\right) .
$$

In the formulas for the connection (A.2) we substitute

$$
\begin{aligned}
\alpha(z, \bar{z}) & =-\frac{1}{2} \log \left(z \bar{z} \log ^{2}(z \bar{z})\right) \\
p(z) & =\frac{a_{-1}}{z}+a_{0}+a_{1} z+\ldots \\
\bar{p}(\bar{z}) & =\frac{a_{-1}}{\bar{z}}+a_{0}+a_{1} \bar{z}+\ldots
\end{aligned}
$$

and solve the section problem in the vicinity of the origin $z=\rho e^{i \phi}, \rho \rightarrow 0$.

At the leading order in $\rho$ we get the following orthonormal pair of solutions

$$
\begin{aligned}
& \hat{\psi}_{1}=\frac{c}{\sqrt{\log (z \bar{z})}}\left(\begin{array}{l}
1 \\
\zeta
\end{array}\right) \\
& \hat{\psi}_{2}=\frac{1}{c \sqrt{\log (z \bar{z})}}\left(\begin{array}{c}
-\frac{\log z}{\zeta} \\
\log \bar{z}
\end{array}\right) .
\end{aligned}
$$

We define the monodromy as

$$
\hat{\psi}_{a}\left(z e^{2 \pi i}\right)=\Omega_{a}^{b} \hat{\psi}_{b}(z)
$$

and see that

$$
\Omega=\left(\begin{array}{cc}
1 & 0 \\
-\frac{2 \pi i}{\zeta c^{2}} & 1
\end{array}\right)
$$

Note that the monodromy is independent of the polynomial, as expected. This monodromy, found near the origin, is the same on the whole complex plane. By choosing the normalization constant to be

$$
c^{2}(\zeta)=-\frac{2 \pi i}{\zeta q}
$$


we get the monodromy that we used in the main body of the text

$$
\Omega(\zeta)=\left(\begin{array}{ll}
1 & 0 \\
q & 1
\end{array}\right)
$$

\section{Appendix B. $Z_{m}$ symmetric polygons}

Let's consider the scattering amplitudes problem in the case of most general $\mathrm{Z}_{\mathrm{m}}$ symmetric polygon. In terms of modified Sinh-Gordon it is given by the following problem

$$
\begin{aligned}
\partial_{z} \partial_{\bar{z}} \alpha(z, \bar{z}) & -e^{2 \alpha(z, \bar{z})}+|p(z)|^{2} e^{-2 \alpha(z, \bar{z})}=0 \\
p(z) & =m^{2} z^{m-2}\left(1+a_{0} z^{m}+\ldots+a_{n-2} z^{m(n-1)}\right) \\
\alpha & \text { regular, } \quad z \rightarrow 0 \\
\hat{\alpha} & \rightarrow 0, \quad|w| \rightarrow \infty
\end{aligned}
$$

Now we can make conformal transformation $z=\tilde{z}^{1 / m}$ which allows us to focus on the one period. We get

$$
\begin{aligned}
\partial_{z} \partial_{\bar{z}} \alpha(z, \bar{z}) & -e^{2 \alpha(z, \bar{z})}+|p(z)|^{2} e^{-2 \alpha(z, \bar{z})}=0 \\
p(z) & =\frac{1}{z}+a_{0}+\ldots+a_{n-3} z^{n-3}+a_{n-2} z^{n-2} \\
\alpha & =l \log (z \bar{z})+\text { regular, } \quad z \rightarrow 0 \\
\hat{\alpha} & \rightarrow 0, \quad|w| \rightarrow \infty \\
l & =\frac{1}{2 m}-\frac{1}{2}
\end{aligned}
$$

here $l=\frac{1}{2 m}-\frac{1}{2}$. This makes a connection with the problem considered recently by Lukyanov and Zamolodchikov [29]. In particular they argue that for this kind of problem there exist smooth limit $l \rightarrow-\frac{1}{2}$ which is

$$
\begin{aligned}
\partial_{z} \partial_{\bar{z}} \alpha(z, \bar{z}) & -e^{2 \alpha(z, \bar{z})}+|p(z)|^{2} e^{-2 \alpha(z, \bar{z})}=0 \\
p(z) & =\frac{1}{z}+a_{0}+\ldots+a_{n-3} z^{n-3}+a_{n-2} z^{n-2} \\
\alpha & =-\frac{1}{2} \log \left(z \bar{z} \log ^{2}(z \bar{z})\right)+\text { less singular, } \quad z \rightarrow 0 \\
\hat{\alpha} & \rightarrow 0, \quad|w| \rightarrow \infty
\end{aligned}
$$

and corresponds to operator insertion problem considered above. 


\section{Appendix C. Computation of $A_{c u t-o f f}$ for $n$ odd}

We are interested in the calculation of the integral

$$
A_{c u t-o f f}=4 \int_{\Sigma_{0}, z_{A d S}>\epsilon} d^{2} w .
$$

The algorithm was first developed in [11], to which we refer the reader for notation and further discussion. Here we just repeat the analysis keeping in mind that we have non-trivial monodromy and check that we get essentially the same as in [11].

Below we think about any flat section as $2 \times 2$ matrix with unit determinant $\psi_{\alpha a}$ where $\alpha$ is inner $S L(2, R)$ and $a$ target $S L(2, R)$ indices.

If we consider $z$ plane with $\psi_{\zeta}(z)$ being exact solution of the problem then the target space solution is

$$
Z=\psi_{\zeta=1}^{T} U \psi_{\zeta=i} .
$$

As we go around the $z$-plane $z \rightarrow e^{2 \pi i} z$ the solution transforms as

$$
\begin{aligned}
& Z \rightarrow \hat{\Omega} Z \hat{\Omega}^{T} \\
& \hat{\Omega}=\left(\begin{array}{ll}
1 & 0 \\
q & 1
\end{array}\right)
\end{aligned}
$$

this monodromy corresponds to the translation of the Poincare coordinate $x \rightarrow x+q$. Recall that having $q$ in lower left position is important. Otherwise the monodromy does not correspond to $x \rightarrow x+q$. This will be important below.

Thus, we have

$$
\psi\left(z e^{2 \pi i}\right)=\psi(z) \hat{\Omega}^{T}
$$

As in [11], we go to the $w$-plane with the gauge transformation being not single-valued in the $z$-plane

$$
\hat{\psi}(w)=g(z) \psi(z) .
$$

As $z \rightarrow e^{2 \pi i} z, w \rightarrow e^{i \pi n} w$ and we have

$$
\hat{\psi}\left(w e^{i \pi n}\right)=e^{i \frac{\pi}{2}(n-2) \sigma_{2}} \hat{\psi}(w) \hat{\Omega}^{T}
$$

for the details see [11] the only difference is the appearance of the $\hat{\Omega}^{T}$ which was the identity matrix for the case of amplitudes. 
The exact solution is smooth. However, as we go to the large $w(\hat{\alpha} \rightarrow 0)$ region the exact solution is approximately equal (in the sense of asymptotic expansion) to (for the left problem)

$$
\hat{\psi}_{\text {appr }}(w)=\left(\begin{array}{cc}
b_{11} e^{w+\bar{w}} & b_{12} e^{w+\bar{w}} \\
b_{21} e^{-w-\bar{w}} & b_{22} e^{-w-\bar{w}}
\end{array}\right)
$$

where coefficients $b_{i j}$ are different in each Stokes sector. For the given Stokes sector we denote them as $b_{i j}^{k}$ where $k$ is the number of the Stokes sector.

If we analytically continue $\hat{\psi}_{\text {appr }}(w)$ as soon as we cross a Stokes line it is not the right form of the asymptotic form of the exact solution. Every time we cross a Stokes line the coefficients of asymptotic expansion jump and this information is encoded in Stokes matrices.

Let's consider

$$
\hat{\psi}_{a p p r}^{n+1}(w)=\left(\begin{array}{cc}
b_{11}^{n+1} e^{w+\bar{w}} & b_{12}^{n+1} e^{w+\bar{w}} \\
b_{21}^{n+1} e^{-w-\bar{w}} & b_{22}^{n+1} e^{-w-\bar{w}}
\end{array}\right)
$$

which corresponds to the correct asymptotic form of the solution in $(n+1)$-th Stokes sector.

Now let's take the large $w$ limit in the (C.6). We get for odd $n$

$$
\hat{\psi}_{a p p r}^{n+1}(-w)=e^{i \frac{\pi}{2}(n-2) \sigma_{2}} \hat{\psi}_{a p p r}^{1}(w) \hat{\Omega}^{T} .
$$

From this equation one can get the relation between $b_{i j}^{n+1}$ and $b_{i j}^{1}$. For the right problem all formulae are completely the same.

The central objects for the computation are $\delta u_{i}$ and $\delta v_{i}$ in [11]. In our notations they are defined as follows

$$
\begin{aligned}
& \delta u_{2 k+1}=-\log \left(b_{11}^{2 k+1} \tilde{b}_{11}^{2 k+1}\right), \quad \delta u_{2 k}=-\log \left(b_{21}^{2 k} \tilde{b}_{21}^{2 k}\right), \\
& \delta v_{2 k+1}=-\log \left(-b_{21}^{2 k+1} \tilde{b}_{11}^{2 k+1}\right), \quad \delta v_{2 k}=-\log \left(b_{11}^{2 k} \tilde{b}_{21}^{2 k}\right) .
\end{aligned}
$$

From the (C.9) both for amplitudes and form factor one gets

$$
\begin{aligned}
\delta u_{n+1} & =\delta u_{1} \\
\delta v_{n+1} & =\delta v_{1} .
\end{aligned}
$$

Equations (C.11) are the same as in the case of amplitudes. Thus, we have exactly the same formula for the area as in the case of amplitudes in terms of $\left(\delta u_{i}, \delta v_{i}\right)$.

After noticing the fact that the formula contains only coordinate difference between two consecutive cusps we conclude that the formula for $A_{\text {cut-of } f}$ part in terms of $l_{i}^{+}$and $l_{j}^{-}$for the form factor is completely identical to the amplitudes' one. Except that instead of $x_{n}^{ \pm}=x_{0}^{ \pm}$we have $x_{n}^{ \pm}=x_{0}^{ \pm}+q$ which does not affect the fact that $l_{i+n}^{ \pm}=l_{i}^{ \pm}$. The final formula is given by $A_{B D S-l i k e}$ in equation (5.8) in [11]. 


\section{Appendix D. Integral equations for complex masses}

Starting from the case of real masses we can introduce phases which modifies the form of integral equations.

For $m_{s}=\left|m_{s}\right| e^{i \phi_{s}}, \quad \bar{m}=|\bar{m}| e^{i \bar{\phi}}$ we have

$$
\begin{aligned}
K_{s, s^{\prime}}(\theta) & =\frac{1}{2 \pi \cosh \left(\theta-\theta^{\prime}+i \phi_{s}-i \phi_{s^{\prime}}\right)} \\
\tilde{Y}_{s}(\theta) & =Y_{s}\left(\theta+i \phi_{s}\right), \quad \tilde{\bar{Y}}=\bar{Y}(\theta+i \bar{\phi})
\end{aligned}
$$

and for $\left|\phi_{s}-\phi_{s+1}\right|<\frac{\pi}{2}$ we have

$$
\begin{aligned}
\log \tilde{Y}_{s} & =-\left|m_{s}\right| \cosh \theta+K_{s, s+1} \star \log \left(1+\tilde{Y}_{s+1}\right) \\
& +K_{s, s-1} \star \log \left(1+\tilde{Y}_{s-1}\right), \quad s=1, \ldots, n-3 \\
\log \tilde{Y}_{n-2} & =-\left|m_{n-2}\right| \cosh \theta+K_{n-2, n-3} \star \log \left(1+\tilde{Y}_{n-3}\right) \\
& +2 K_{n-2, \bar{\phi}} \star \log (1+\tilde{\bar{Y}}) \\
\log \tilde{\bar{Y}} & =-|\bar{m}| \cosh \theta+K_{n-2, \bar{\phi}} \star \log \left(1+\tilde{Y}_{n-2}\right)
\end{aligned}
$$

as soon as we cross the lines $\left|\phi_{s}-\phi_{s+1}\right|=\frac{\pi}{2}, \frac{3 \pi}{2}, \ldots$ we get the contribution from the pole in the kernel and the form of integral equation will change. This was discussed in detail in [13].

\section{Appendix E. Calculation of $N=4 k$ gluon amplitudes by taking the double soft limit}

Let us consider the amplitude for $2 n$ gluons with $n$ being odd and then take the double soft limit to get $2(n-1)$ gluons amplitude. This step is necessary since we have derived the full expression for the area only for $n$ odd. This is a quick way to get the result when $n$ is even.

Here we present formulas for the area which depend only on the cross ratios. If we introduce the new variables

$$
\begin{aligned}
X_{2 k}(\theta) & =Y_{2 k}(\theta) \\
X_{2 k+1}(\theta) & =Y_{2 k+1}\left(\theta-i \frac{\pi}{2}\right)=Y_{2 k+1}^{-} \\
X_{n-1}(\theta) & =\bar{Y}(\theta)
\end{aligned}
$$


We choose integration contours $l_{\gamma}$ in a canonical way so that all $Z_{\gamma} / e^{\theta^{\prime}}$ are real and negative

$$
\begin{aligned}
l_{2 k} & =\left(i \phi_{2 k}-\infty, i \phi_{2 k}+\infty\right) \\
l_{2 k+1} & =\left(i\left(\phi_{2 k+1}+\frac{\pi}{2}\right)-\infty, i\left(\phi_{2 k+1}+\frac{\pi}{2}\right)+\infty\right) \\
l_{n-1} & =(i \bar{\phi}-\infty, i \bar{\phi}+\infty)
\end{aligned}
$$

we get that the Y-system can be rewritten as follows

$$
\log X_{s}(\theta)=Z_{s} e^{-\theta}+\bar{Z}_{s} e^{\theta}+\frac{1}{2 \pi i} \sum_{r=1}^{n-1} \Omega(r) \theta^{s r} \int_{l_{r}} \frac{d \theta^{\prime}}{\sinh \left(\theta^{\prime}-\theta\right)} \log \left(1+X_{r}\left(\theta^{\prime}\right)\right)
$$

where $\theta^{s r} 9$ is the intersection form. Or, using the notation of [8],

$$
\log X_{s}(\theta)=Z_{s} e^{-\theta}+\bar{Z}_{s} e^{\theta}+\frac{1}{2 \pi i} \sum_{s^{\prime}=1}^{n-1} \Omega\left(s^{\prime}\right)\left\langle s, s^{\prime}\right\rangle \int_{l_{s^{\prime}}} \frac{d \theta^{\prime}}{\sinh \left(\theta^{\prime}-\theta\right)} \log \left(1+X_{s^{\prime}}\left(\theta^{\prime}\right)\right)
$$

with $\langle s, s+1\rangle=-\langle s+1, s\rangle=(-1)^{s+1}$. Here $Z_{2 k}=-\frac{m_{2 k}}{2}, Z_{2 k+1}=-i \frac{m_{2 k+1}}{2}$.

The case of amplitudes then corresponds to 10

$$
\Omega(s)=1, \quad s=1, . ., n-3
$$

and the case of the form factor to

$$
\begin{aligned}
\Omega(s) & =1, \quad s=1, \ldots, n-2 \\
\Omega(n-1) & =2 .
\end{aligned}
$$

The full answer for the area then takes the form

$$
A=A_{d i v}+A_{B D S-l i k e}+A_{0}+Y Y_{c r}+C_{0}
$$

here $C^{0}$ is the constant which comes from the difference between $A_{S i n h}$ and $A_{f r e e}$ and is given

$$
\begin{aligned}
\text { Scattering Amplitudes : } & C_{0}^{s a}=\frac{7 \pi}{12}(n-2) \\
\text { Form Factor : } & C_{0}^{f f}=\frac{3 \pi}{4}+\frac{7 \pi}{12}(n-1) .
\end{aligned}
$$

\footnotetext{
$9 \theta^{s r}=(-1)^{s+1}\left(\delta_{s+1, r}+\delta_{s-1, r}\right)$

10 This $\Omega$ should not be confused with the monodromy $\Omega$.
} 
$Y Y_{c r}$ is the critical value of the Yang-Yang functional that depends only on Y-functions. $A_{d i v}$ and $A_{B D S-l i k e}$ are known and can be found in [1] (equation 5.8).

Remember that the phases of the mass parameters entered in the equations for the area as a choice of contours of integration. To get rid of masses completely we would like to go to the region where all masses parameters are big $m_{s}>>1$ and where there is a simple relation between the masses and the physical cross ratios. We define $\hat{m}_{s}$ via $Z_{s}=-\hat{m}_{s} / 2$ $\left(\hat{m}_{2 k}=m_{2 k}, \hat{m}_{2 k+1}=i m_{2 k+1}\right)$. We write $\hat{m}_{s}=\left|\hat{m}_{s}\right| e^{i \hat{\phi}_{s}}$

$$
\log \chi_{s}^{+}=-\left|\hat{m}_{s}\right| \cos \hat{\phi}_{s}, \quad \log \chi_{s}^{-}=-\left|\hat{m}_{s}\right| \sin \hat{\phi}_{s}
$$

We choose $0<\hat{\phi}_{2 k+1}<\frac{\pi}{2}$ and $-\frac{\pi}{2}<\hat{\phi}_{2 k}<0$. With this choice we do not cross any poles when we express masses through physical cross ratios.

Now we want to take the double soft limit. We fix all phases and send $\left|\hat{m}_{1}\right| \rightarrow \infty$. This corresponds to $x_{-1}^{ \pm}-x_{0}^{ \pm}=\epsilon^{ \pm} \rightarrow 0$. In this case $\chi_{1}^{ \pm} \rightarrow 0$ equivalently $X_{1}(\zeta) \rightarrow 0$.

When we take the double soft limit, the amplitude $A-A_{\text {div }}$ behaves in a known way

$$
A^{2 n}\left(\ldots, x_{-1}^{ \pm}, x_{0}^{ \pm}, \ldots\right) \rightarrow \frac{1}{4} \log \frac{\epsilon^{+}}{x_{01}^{+}} \log \frac{\epsilon^{-}}{x_{-20}^{-}}+\frac{7 \pi}{12}+A^{2 n-2}\left(\ldots, x_{0}^{ \pm}, \ldots\right) .
$$

we write explicitly $\frac{7 \pi}{12}$ due to the fact that as one of the masses goes to infinity we get the contribution from the isolated zero to the finite part of the answer which is not related to the amplitude we are interested in.

We rewrite the amplitude as

$$
A=A_{\text {div }}+A_{B D S}+R
$$

where

$$
R=A_{B D S-l i k e}-A_{B D S}+A_{0}+Y Y_{c r}+C_{0}
$$

Then the behavior of (E.10) is totally captured by $A_{B D S}$ while $R$ being the function of only cross ratios has a smooth limit which we will study. Under the soft limit $Y Y_{c r}$ changes trivially simply by setting $Y_{1}$ to zero in the formulas. The same happens with $C_{0}(n) \rightarrow C_{0}(n-1)$ in (E.7).

However for the part $\left(A_{B D S-l i k e}-A_{B D S}\right)+A_{0}$ more interesting things happen. Namely each of two pieces has the part that diverges in the limit but as all divergencies are already captured by $A_{B D S}$ they should cancel.

We will see that the structure of each term is such that allows one to take the limit easily. 


\section{E.1. Soft limit of the $A_{0}$}

Here we discuss the behavior of the $A_{0}$ when we take the soft limit. We have that

$$
\begin{aligned}
A_{0}^{n} & =-\frac{1}{2} \sum_{s, s^{\prime}=1}^{n-3} w_{s, s^{\prime}}^{n} \log \chi_{s}^{+} \log \chi_{s^{\prime}}^{-} \\
& =-\frac{1}{2} \sum_{s^{\prime}=1}^{n-3} w_{1, s^{\prime}}^{n}\left(\log \chi_{1}^{+} \log \chi_{s^{\prime}}^{-}-\log \chi_{s^{\prime}}^{+} \log \chi_{1}^{-}\right)+\tilde{A}_{0}^{n-2} \\
& \tilde{A}_{0}^{n-1}=\tilde{A}_{0}^{n-2}=-\frac{1}{2} \sum_{s, s^{\prime}=1}^{n-5} w_{s, s^{\prime}}^{n-2} \log \chi_{s+2}^{+} \log \chi_{s^{\prime}+2}^{-}
\end{aligned}
$$

where we used that $w_{s, s^{\prime}}=-w_{s^{\prime}, s}, w_{2, s^{\prime}>1}=0$ and that $w_{s+2, s^{\prime}+2}^{n}=w_{s, s^{\prime}}^{n-2}$ where $w_{s, s^{\prime}}^{n-2}$ is the inverse intersection form for $2(n-2)$ gluons problem which exists due to the fact that $(n-2)$ is again odd.

Thus, we see that after taking the soft limit $A_{0}^{n} \rightarrow \tilde{A}_{0}^{n-1}$ as first part cancels the same one from $A_{B D S-l i k e}-A_{B D S}$.

\section{E.2. Soft limit of the $A_{B D S-l i k e}-A_{B D S}$}

As was shown in 11

$$
\Delta A_{B D S}=A_{B D S-l i k e}-A_{B D S}=\frac{1}{4} \sum_{i=1}^{n} \sum_{j=1, j \neq i, i-1}^{n} \log \frac{c_{i, j}^{+}}{c_{j+1, i}^{+}} \log \frac{c_{i-1, j}^{-}}{c_{i, j}^{-}}
$$

where $c_{i, j}=c_{j, i}$ are the uniquely defined cross ratios. They start with the factor $x_{i j}$ in the numerator and are then completed to cross ratios by neighboring differences such as $x_{i, i+1}$. We also define $c_{i, j}=1$ for $|i-j| \leq 1$. For $n$ odd this defines them uniquely. Not all these cross ratios are functionally independent.

When we take the soft limit each $c_{i j}$ can be finite or go to zero or infinity. Each $c_{i j}$ can be rewritten as $c_{i, j}=\chi_{1}^{q_{i, j}} \times \hat{c}_{i j}$ where $q_{i, j}$ is an integer equal to $-1,0,1$ depending on the particular cross ratio. Here $\chi_{1}$ is the cross ratio appearing in our basis of Y-functions that we are sending to zero. $\hat{c}_{i, j}$ are cross ratios which we describe below. Thanks to the logs each term in the sum then can be rewritten as

$$
\begin{aligned}
\log c_{a_{1}, b_{1}}^{+} \log c_{a_{2}, b_{2}}^{-}= & \log \left(\chi_{1}^{+}\right)^{q_{a_{1}, b_{1}}} \log \left(\chi_{1}^{-}\right)^{q_{a_{2}, b_{2}}}+\log \left(\chi_{1}^{+}\right)^{q_{a_{1}, b_{1}}} \log \hat{c}_{a_{2}, b_{2}}^{-} \\
& +\log \hat{c}_{a_{1}, b_{1}}^{+} \log \left(\chi_{1}^{+}\right)^{q_{a_{2}, b_{2}}}+\log \hat{c}_{a_{1}, b_{1}}^{+} \log \hat{c}_{a_{2}, b_{2}}^{-} .
\end{aligned}
$$




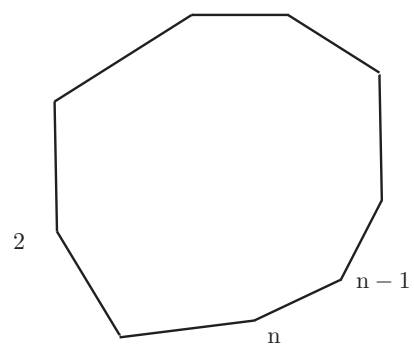

(a)

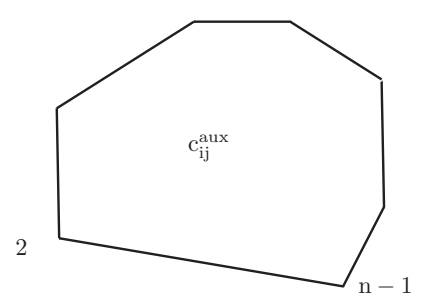

(c)

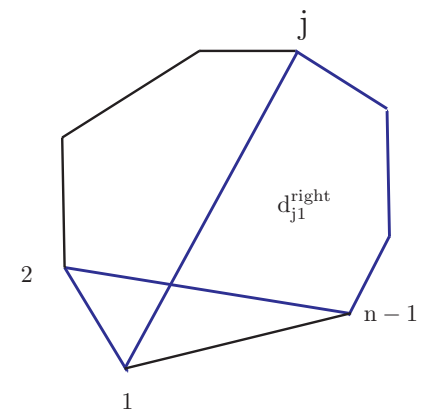

(e)

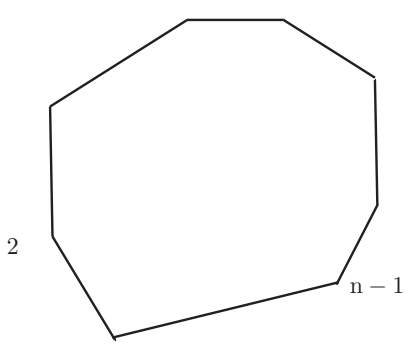

1 (b)

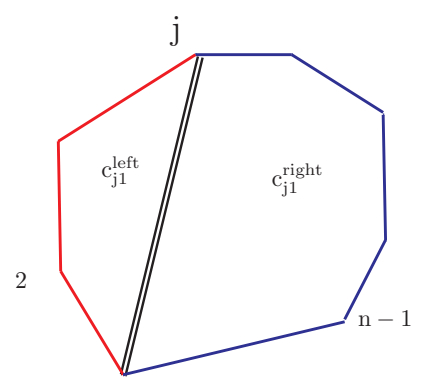

(d)

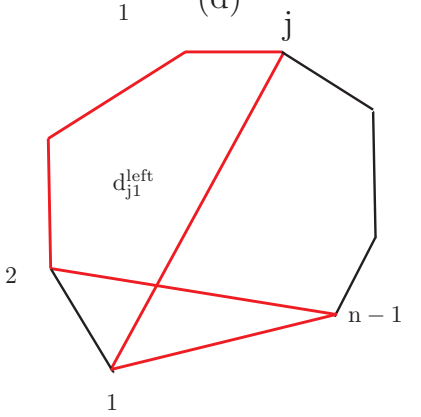

(f)

Fig. 11: In (a) we arrange the $x^{ \pm}$coordinates of the original polygon with $2 n$ cusps and $n$ being odd. Then we take the double soft limit by sending $x_{n}^{ \pm} \rightarrow x_{1}^{ \pm}$. Here we draw only the $x_{i}^{+}$positions, for $x_{i}^{-}$we have the same. The resulting polygon with $2(n-1)$ number of cusps is shown in (b). In (c) we show the auxiliary Wilson polygon with $2(n-2)$ cusps which we use to evaluate $c_{i j}^{a u x}$. They are uniquely defined because $(n-2)$ is odd. In $(\mathrm{d})$ we illustrate the definition of $c_{j 1}^{\text {left }}$ (in red) and of $c_{j 1}^{r i g h t}$ (in blue). In (e) and (f) we illustrate the definition of $d_{j 1}^{l e f t}$ (in red) and of $d_{j 1}^{\text {right }}$ (in blue). For (d-f) the cross ratio is formed by starting with $x_{j 1}^{ \pm}$in the numerator and then following the depicted path, putting the next difference in the denominator, the following in the numerator, etc.

We see that each term in the sum produces a unique finite piece. Divergencies at the same time should cancel among each other and with the terms coming from the $A_{0}$. Thus, under the soft limit $\Delta A_{B D S}^{n} \rightarrow \Delta \tilde{A}_{B D S}^{n-1}$ where

$$
A_{B D S}^{\tilde{n}-1}-A_{B D S-l i k e}^{n-1}=\Delta \tilde{A}_{B D S}^{n-1}=\frac{1}{4} \sum_{i=1}^{n} \sum_{j=1, j \neq i, i-1}^{n} \log \frac{\hat{c}_{i, j}^{+}}{\hat{c}_{j+1, i}^{+}} \log \frac{\hat{c}_{i-1, j}^{-}}{\hat{c}_{i, j}^{-}}
$$


and $\hat{c}_{i-1, j}$ will be defined below without any reference to the original polygon. This is the definition of $A_{B D S-l i k e}^{n-1}$ when $n-1$ is even.

To explain pictorially how they are defined it is useful to change the counting of cusps from $-\frac{n-1}{2}, \ldots, 0, \ldots, \frac{n-1}{2}$ which is convenient when we think in terms of $\mathrm{Y}$-system to $1, . ., n$ with the first cusp corresponding to zeroth one. Then taking the double soft limit corresponds to $x_{n} \rightarrow x_{1}$ and we end up with the polygon labeled by $\left(x_{1}, \ldots, x_{n-1}\right)$.

The definition of the $\hat{c}_{i j}$ is simple in terms of the auxiliary odd cusp polygon which is formed by $\left(x_{2}, \ldots, x_{n-1}\right)$ points. Remember the point $x_{1}$ was special in taking the soft limit. Then $\hat{c}_{i, j}$ with $i, j \neq 1, n$ are just $c_{i j}^{a u x}$ cross ratios which we defined before for the general odd $n$ polygon applied to the auxiliary polygon. See fig. 11 (c).

Next $\hat{c}_{j, 1}$ and $\hat{c}_{n, j}$ with $j$ being even are given be $c_{j, 1}^{\text {left }}$ and $c_{j, 1}^{\text {right }}$ where these are cross ratios defined as in the case of odd polygon but applied for the even polygon that we get after taking the soft limit. For the even polygon there are two cross ratios we can make from a given distance (we can close it on the left or on the right, left or right are defined relative to the vector going from $i$ to $j$ where $i<j$ ). See fig. 11 (d). Notice that $\hat{c}_{n-1,1}=c_{n-1,1}^{\text {left }}=\frac{1}{c_{2,1}^{\text {right }}}=\frac{1}{\hat{c}_{n, 2}}$ being equal to "round" cross ratio that is specific only for $n$ even cases and contain only consecutive cusps.

The last parts are $\hat{c}_{j, 1}$ and $\hat{c}_{n, j}$ with $j$ being odd. These are given by $d_{j, 1}^{\text {right }}$ and $d_{j, 1}^{\text {left }}$. For the definition see fig. 11 (e-f). Their new feature is flip between non-consecutive cusps before closing the loop.

Putting all together we have

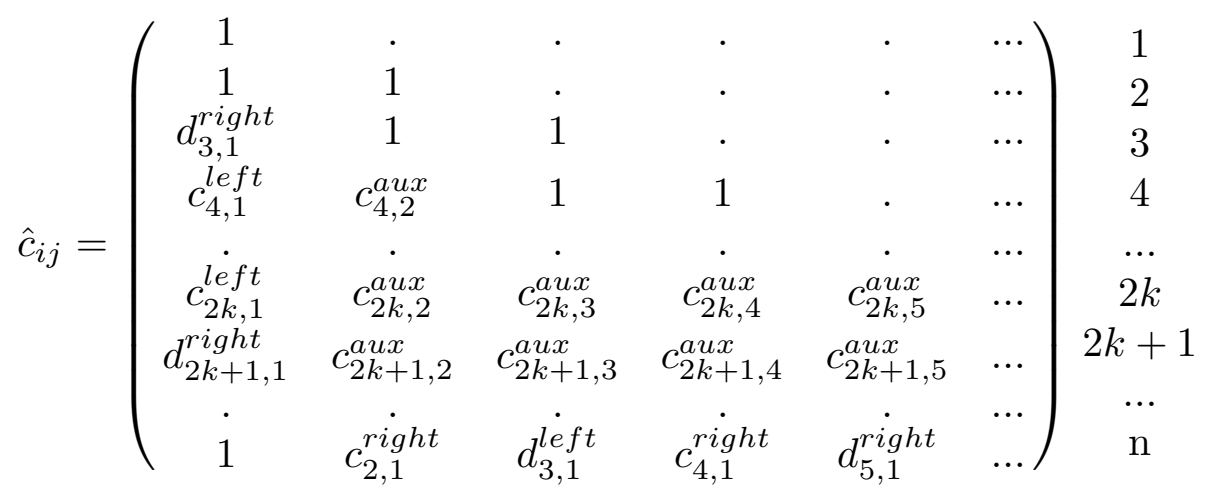




\begin{tabular}{|c|c|}
\hline Case of $2(n-1)$ gluons & $\hat{c}_{i, j}, \quad i>j$ \\
\hline$i, j \neq 1, n$. & $c_{i, j}^{\text {aux }}$ \\
\hline$j=1 ; \quad i=2 k$. & $c_{i, 1}^{\text {left }}=\frac{x_{i, 1} x_{i-1, i-2} \ldots x_{3,2}}{x_{i, i-1} \ldots x_{2,1}}$ \\
\hline$j=1 ; \quad i=2 k+1$. & $d_{i, 1}^{r i g h t}=\frac{x_{i, 1} x_{i+2, i+1} \ldots x_{n-1,2}}{x_{i+1, i} \ldots x_{2,1}}$ \\
\hline$i=n ; \quad j=2 k$. & $c_{j, 1}^{r i g h t}=\frac{x_{j, 1} x_{j+2, j+1} \ldots x_{n-1, n-2}}{x_{j+1, i} \ldots x_{n-1,1}}$ \\
\hline$i=n ; \quad j=2 k+1$. & $d_{j, 1}^{\text {left }}=\frac{x_{j, 1} x_{j-1, j-2} \ldots x_{n-1,2}}{x_{j, j-1} \ldots x_{n-1,1}}$ \\
\hline
\end{tabular}

Table 2: Explicit expressions for the $\hat{c}_{i j}$ cross ratios when $n$ is even.

These are represented graphically in figure fig. 11 and should be inserted in (E.17).

To summarize let's give once more the expression for the full area for the given even $\tilde{n}=n-1$

$$
A=A_{d i v}+A_{B D S}+\Delta \tilde{A}_{B D S}^{\tilde{n}}+\tilde{A}_{0}^{\tilde{n}}+Y Y_{c r}+C_{0}
$$

with $\Delta \tilde{A}_{B D S}^{\tilde{n}}$ given by $(\mathbb{E} .17)$ and $\tilde{A}_{0}^{\tilde{n}}$ by $(\mathbb{E} .14)$ where we have chosen the first cusp as being special. One can symmetrize the expression over all cusps to consider all cusps on an equal footing.

In the case of form factors there is slight difference in taking the soft limit which is due to the fact that anomalous conformal Ward identity 35] acts on the infinite number of points of the periodic polygon and we have no analogue of $A_{B D S}$ which solves it and is defined over one period. In that case we take the soft limit directly at the level of $A_{B D S-l i k e}$ all the rest is completely the same.

\section{E.3. The octagon}

Let's apply formulas given above to get the answer for the octagon $n=4$ as a soft limit from the decagon $n=5$. A lot of simplifications occur in this first non-trivial case for the amplitudes.

First, notice that $\tilde{A}_{0}$ is equal to zero in this case.

As a next step let's analyze the $\Delta \tilde{A}_{B D S}$. Because of few number of cusps there is no possibility to form a cross ratios using auxiliary Wilson polygon. Thus, the $\hat{c}_{i j}$ take the following form

$$
\hat{c}_{i j}=\left(\begin{array}{ccccc}
1 & \cdot & \cdot & \cdot & \cdot \\
1 & 1 & \cdot & \cdot & \cdot \\
d_{3,1}^{\text {right }} & 1 & 1 & \cdot & \cdot \\
c_{4,1}^{\text {left }} & 1 & 1 & 1 & \cdot \\
1 & c_{2,1}^{\text {right }} & d_{3,1}^{\text {left }} & 1 & 1
\end{array}\right)
$$


In terms of the cross ratio $\chi$ defined in [11] these are

$$
\begin{aligned}
d_{3,1}^{\text {right }} & =1+\chi, \quad d_{3,1}^{\text {left }}=\frac{1+\chi}{\chi} \\
c_{4,1}^{\text {left }} & =\frac{1}{c_{2,1}^{\text {right }}}=\chi
\end{aligned}
$$

plugging it to the formula (E.17) one gets that $\Delta \tilde{A}_{B D S}=-\frac{1}{2} \log \left(1+\chi^{-}\right) \log \left(1+\frac{1}{\chi^{+}}\right)$.

The next piece is the critical value of Yang-Yang functional. In the case of $n=4$ the solution of the Y-system is simply $X_{1}(\zeta)=e^{Z_{1} / \zeta+\bar{Z}_{1} \zeta}$. Thus, we have

$$
\begin{aligned}
Y Y_{c r} & =-\frac{1}{2 \pi} \int_{l_{1}} d \theta \frac{\cosh 2 \theta}{\sinh 2 \theta}\left(\sinh \theta \log \chi^{+}-i \cosh \theta \log \chi^{-}\right) \log \left(1+X_{1}(\theta)\right) \\
& =\int_{-\infty}^{\infty} d t \frac{|m| \sinh t}{2 \pi \tanh (2 t+2 i \phi)} \log \left(1+e^{-2|m| \cosh t}\right), \quad \phi \in\left(-\frac{\pi}{2}, 0\right)
\end{aligned}
$$

with

$$
\begin{aligned}
& \log \chi^{+}=-\log \chi_{2}^{+}=|m| \cos \phi \\
& \log \chi^{-}=-\log \chi_{2}^{-}=|m| \sin \phi .
\end{aligned}
$$

The last step is $C_{0}(4)=\frac{7 \pi}{6}$ which finishes the calculation of the area for the octagon. Notice that to get the answer in the form of [11] we should make change of variables $\phi=\tilde{\phi}-\frac{\pi}{2}$.

\section{Appendix F. On the connection with BTZ black holes}

The cases which we have considered are very special cases of quotients of $A d S_{3}$ by particular group element. All these are very special cases of BTZ black holes 1 which are the solutions of 3D Einstein gravity

$$
\begin{aligned}
& d s^{2}=-\left(r^{2}-M\right) d \tau^{2}-J d t d \phi+\frac{d r^{2}}{r^{2}-M+\frac{J}{4 r^{2}}}+r^{2} d \phi^{2} \\
& 0 \leq \phi \leq 2 \pi
\end{aligned}
$$

where we set $R=1$.

If we set $J=0$ and $M=-\frac{1}{m^{2}} 12$ this geometry correspond to $A d S_{3}$ with the identification $\phi \sim \phi+\frac{2 \pi}{m}$. In the case of $m=\infty$ or $M=0$ this is the Poincare metric of

11 We are grateful to Benjamin Basso for pointing this out.

12 Although these solution have naked singularity we call them BTZ black holes in generalized sense [36]. 
$A d S_{3}$ with $\phi$ being spatial dimension and the identification $\phi \sim \phi+2 \pi$ which appears in the form factor problem. In the case $m=1$ this is the $A d S_{3}$ metric which appears in the amplitude problem.

One can consider the black holes with non-zero angular momenta and positive masses. However the physical interpretation of these solutions in terms of field theory is not clear for us.

\section{Appendix G. The monodromy in terms of coordinates}

Here we write formulas for $x^{+}$. Formulas for $x^{-}$are completely the same. As was explained in the text we can consider points $x_{i}^{+}$with $i \in[0, n+2]$. Then we can use conformal transformation to fix $x_{0}^{+}, x_{1}^{+}, x_{2}^{+}$. The monodromy relates coordinate of point $x_{i+n}^{+}$ to $x_{i}^{+}$. Thus, we can express the components of the monodromy in terms of coordinates $\left(x_{0}^{+}, x_{1}^{+}, x_{2}^{+}, x_{n}^{+}, x_{n+1}^{+}, x_{n+2}^{+}\right)$. To do it we solve the following system of equations

$$
\begin{aligned}
x_{i+n}^{+} & =\frac{\hat{\Omega}_{11}+\hat{\Omega}_{12} x_{i}^{+}}{\hat{\Omega}_{21}+\hat{\Omega}_{22} x_{i}^{+}}, \quad i=0,1,2 \\
\operatorname{det}[\hat{\Omega}] & =1
\end{aligned}
$$

where as usually when it is possible we enumerate the cusps in such a way that $x_{i+1}^{+}>x_{i}^{+}$. The solution is

$$
\begin{aligned}
A & =x_{1,0}^{+} x_{2,0}^{+} x_{2,1}^{+} x_{n+1, n}^{+} x_{n+2, n}^{+} x_{n+2, n+1}^{+} \\
\hat{\Omega}_{11} & =\frac{1}{\sqrt{A}}\left(x_{0}^{+} x_{1}^{+} x_{n+1, n}^{+}+x_{1}^{+} x_{2}^{+} x_{n+2, n+1}^{+}-x_{0}^{+} x_{2}^{+} x_{n+2, n}^{+}\right) \\
\hat{\Omega}_{22} & =\frac{1}{\sqrt{A}}\left(x_{n}^{+} x_{n+1}^{+} x_{1,0}^{+}+x_{n+1}^{+} x_{n+2}^{+} x_{2,1}^{+}-x_{n}^{+} x_{n+2}^{+} x_{2,0}^{+}\right) \\
\hat{\Omega}_{12} & =\frac{1}{\sqrt{A}}\left(x_{2}^{+} x_{n+1, n}^{+}+x_{0}^{+} x_{n+2, n+1}^{+}-x_{1}^{+} x_{n+2, n}^{+}\right) \\
\hat{\Omega}_{21} & =\frac{1}{\sqrt{A}}\left(-x_{0}^{+} x_{n+1}^{+} x_{n+2}^{+} x_{2,1}^{+}+x_{1}^{+} x_{n}^{+} x_{n+2}^{+} x_{2,0}^{+}-x_{2}^{+} x_{n}^{+} x_{n+1}^{+} x_{1,0}^{+}\right) .
\end{aligned}
$$

As the monodromy is defined up to a sign we choose the solution by claiming that the case $x_{i+n}^{+}=x_{i}^{+}$corresponds to the unit monodromy.

The trace of the monodromy is equal to

$$
\operatorname{Tr}[\hat{\Omega}]=\frac{1}{\sqrt{A}}\left(x_{n+2,0}^{+} x_{n+1, n}^{+} x_{2,1}^{+}-x_{1,0}^{+} x_{n, 2}^{+} x_{n+2, n+1}^{+}\right) .
$$

Notice that while the separate components of the monodromy are not conformal invariant the trace is as it should be. The monodromy $\hat{\Omega}$ in this section corresponds to $\hat{\Omega}(\zeta=1)$ in the main body of the text. For the $x^{-}$coordinates it would be $\hat{\Omega}(\zeta=i)$. 


\section{References}

[1] L. F. Alday and J. M. Maldacena, JHEP 0706, 064 (2007) [arXiv:0705.0303 [hep-th]].

[2] J. M. Drummond, G. P. Korchemsky and E. Sokatchev, Nucl. Phys. B 795, 385 (2008) arXiv:0707.0243 [hep-th]].

[3] A. Brandhuber, P. Heslop and G. Travaglini, Nucl. Phys. B 794, 231 (2008) arXiv:0707.1153 [hep-th]].

[4] Z. Bern, L. J. Dixon, D. A. Kosower, R. Roiban, M. Spradlin, C. Vergu and A. Volovich, Phys. Rev. D 78, 045007 (2008) [arXiv:0803.1465 [hep-th]].

[5] C. Anastasiou, A. Brandhuber, P. Heslop, V. V. Khoze, B. Spence and G. Travaglini, JHEP 0905, 115 (2009) [arXiv:0902.2245 [hep-th]].

[6] V. Del Duca, C. Duhr and V. A. Smirnov, JHEP 1005, 084 (2010) arXiv:1003.1702 [hep-th]].

[7] L. F. Alday and J. Maldacena, JHEP 0711, 068 (2007) [arXiv:0710.1060 [hep-th]].

[8] L. F. Alday, D. Gaiotto, J. Maldacena, A. Sever and P. Vieira, arXiv:1006.2788 [hepth].

[9] L. F. Alday, B. Eden, G. P. Korchemsky, J. Maldacena and E. Sokatchev, arXiv:1007.3243 [hep-th].

[10] B. Eden, G. P. Korchemsky and E. Sokatchev, arXiv:1007.3246 [hep-th].

[11] L. F. Alday and J. Maldacena, JHEP 0911, 082 (2009) [arXiv:0904.0663 [hep-th]].

[12] L. F. Alday, D. Gaiotto and J. Maldacena, arXiv:0911.4708 [hep-th].

[13] L. F. Alday, J. Maldacena, A. Sever and P. Vieira, arXiv:1002.2459 [hep-th].

[14] C. N. Yang and C. P. Yang, J. Math. Phys. 10, 1115 (1969).

[15] A. B. Zamolodchikov, Nucl. Phys. B 342, 695 (1990).

[16] D. Gaiotto, G. W. Moore and A. Neitzke, arXiv:0807.4723 [hep-th].

[17] D. Gaiotto, G. W. Moore and A. Neitzke, arXiv:0907.3987 [hep-th].

[18] V. A. Kazakov, A. Marshakov, J. A. Minahan and K. Zarembo, JHEP 0405, 024 (2004) arXiv:hep-th/0402207.

[19] N. Dorey and B. Vicedo, JHEP 0607, 014 (2006) arXiv:hep-th/0601194.

[20] M. Banados, C. Teitelboim and J. Zanelli, Phys. Rev. Lett. 69, 1849 (1992) arXiv:hepth/9204099].

[21] W. L. van Neerven, Z. Phys. C 30, 595 (1986).

[22] J. Polchinski and M. J. Strassler, JHEP 0305, 012 (2003) arXiv:hep-th/0209211].

[23] D. M. Hofman and J. Maldacena, JHEP 0805, 012 (2008) arXiv:0803.1467 [hep-th]].

[24] G. Yang, arXiv:1004.3983 [hep-th].

[25] K. Pohlmeyer, Commun. Math. Phys. 46, 207 (1976).

[26] H. J. De Vega and N. G. Sanchez, Phys. Rev. D 47, 3394 (1993).

[27] N. J. Hitchin, Proc. Lond. Math. Soc. 55, 59 (1987). 
[28] A.N.Kirillov, Journal of Mathematical Sciences, Volume 47, Number 2 / October, 1989

[29] S. L. Lukyanov and A. B. Zamolodchikov, JHEP 1007, 008 (2010) arXiv:1003.5333 [math-ph]].

[30] B. M. McCoy, C. A. Tracy and T. T. Wu, J. Math. Phys. 18, 1058 (1977).

[31] A. B. Zamolodchikov, Nucl. Phys. B 432, 427 (1994) arXiv:hep-th/9409108.

[32] A. B. Zamolodchikov, Phys. Lett. B 253, 391 (1991).

[33] P. Fendley, Nucl. Phys. B 374, 667 (1992) arXiv:hep-th/9109021.

[34] S. S. Gubser, I. R. Klebanov and A. M. Polyakov, Nucl. Phys. B 636, 99 (2002) arXiv:hep-th/0204051.

[35] J. M. Drummond, J. Henn, G. P. Korchemsky and E. Sokatchev, Nucl. Phys. B 826, 337 (2010) [arXiv:0712.1223 [hep-th]].

[36] A. R. Steif, Phys. Rev. D 53, 5521 (1996) arXiv:hep-th/9504012]. 